\title{
Nanotechnologies to enable high-performance superconductors for energy applications
}

\author{
C. Cantoni and A. Goyal \\ Oak Ridge National Laboratory, P.O. Box 2008, Oak Ridge, Tennessee \\ 37831, USA
}

\section{Abstract}

High Temperature Superconductors (HTS) wires or coated conductors (CC) hold promise to revolutionize the transmission of electricity enabling the present electric grid to meet the world's growing energy needs. Although superconducting wires can carry 150 times more power than copper wires of the same cross section, further performance improvements are necessary for the superconducting technology to become costcompetitive. This objective can be achieved by introducing and controlling nano-sized defects and non-superconducting phases within the superconducting film's matrix. Such nanostructures, when carefully engineered, significantly increase the loss-free current sustained by the superconductor through a mechanism known as flux pinning. This chapter is a review of the various types of nanostructures that are artificially introduced in superconducting films to enhance the superconductor's performance. Different approaches, materials, and techniques are discussed and the most recent results in this field compared. The last section of this chapter discusses an additional example of 
nanotechnology employment in superconducting wires. This nanotechnology can be regarded as an atomic surface treatment designed to enable the right crystallographic orientation of the superconducting film deposited on the metal template.

\section{Overcoming limitations to superconductors' performance}

Efficient transport and storage of energy are two key factors in the formidable quest to meet the world's growing energy needs. Because electricity is the energy form of choice for most uses, and the most effective way to transport energy from and to different locations, addressing the energy problem means necessarily addressing the shortcomings of the present electric power grid. Capacity and reliability of the present grid are entirely inadequate to accommodate an expected growth in energy demand of $50 \%$ by the year 2030, most of which will be concentrated in urbanized areas whose infrastructures are already severely congested. In addition, $7-10 \%$ of the electricity generated is lost in the grid by heat dissipation, and transmission limitations have already caused black outs throughout the world. Superconducting cables made with high critical temperature $\left(T_{c}\right)$ cuprate superconductors have the potential to transform the grid to meet the $21^{\text {st }}$ century demands. Such cables can carry five times more power then copper cables with the same cross section and exhibit considerable lower losses (loss-free dc transmission). Because lower losses enable longer transmission lengths, superconductors provide the best choice for building a new, non-fragmented, super grid that will enable massive long-distance power transmission, interconnect entire continents, and provide widespread, local energy storage. In such a grid, renewable source power plants in the most remote areas can deliver power anywhere in the continent according to real time demands, and episodic 
surpluses are readily stored for future use. High temperature superconducting (HTS) cables can in fact be exploited in conjunction with solar and wind power for building "green power superhighways" or used to serve congested urban agglomerates replacing copper in existing underground transmission conduits. In addition, HTS can provide oilfree transformers, compact motors and generators, superconducting magnetic-energy storage (SMES) units, and fault current limiters, all with much improved efficiency and reliability and more environmentally friendly as compared to the present infrastructure. All these applications require that the high $\mathrm{T}_{\mathrm{c}}$ superconductor of choice, $\mathrm{YBa}_{2} \mathrm{Cu}_{3} \mathrm{O}_{7-\mathrm{x}}$ (YBCO), be formed into long and flexible conductors that can replace copper wires. By itself, this is already a formidable obstacle because all high $\mathrm{T}_{\mathrm{c}}$ superconductors are brittle ceramics, and the large anisotropy of their physical and transport properties requires that all the crystallites in the conductors be very closely aligned along the three principle crystal axes, with the current flowing in the basal $(a b)$ plane. Only ten years ago, there were still serious doubts about whether YBCO could be shaped in the form of kilometerslong wires with the required performance for commercial applications. This doubt has now been lifted thanks to tremendous advances in nanotechnology and the consequent ability to control and engineer oxide/metal interfaces and multilayers of various thicknesses. Successful technology partnerships between research institutions and industrial companies has allowed for the newest scientific achievements to be promptly transferred from the laboratory to large-scale production. Currently, HTS wires are fabricated using the second generation (2G), also called coated conductors (CC) wire technology. They utilize YBCO or similar cuprate oxides with the chemical formula $\mathrm{RBa}_{2} \mathrm{Cu}_{3} \mathrm{O}_{7-\mathrm{x}}$ where $\mathrm{R}$ is a rare earth or a mixture of rare-earth ions $(\mathrm{Nd}, \mathrm{Sm}, \mathrm{Gd}, \mathrm{Dy}$, 
etc.). The superconductor is processed in the form of an epitaxial film deposited by different methods on a near single-crystal flexible template comprising a metal substrate and epitaxial oxide buffer layers. The template is manufactured using two major technologies: the rolling assisted biaxially textured substrate (RABiTS) technology [1-3], and the ion beam assisted deposition (IBAD) technology [4-6]. As shown in Fig.1, the RABiTS approach relies on producing a biaxially textured Ni-5\%W alloy template on which oxide buffer layers and the superconductor are epitaxially deposited. In the IBAD approach, the metal alloy substrate is polycrystalline and the biaxial texture is developed in an $\mathrm{MgO}$ thin film by the assisted ion beam deposition, and then transferred by epitaxy to the subsequent layers. Wires of 100-500 meters in length carrying currents of over 300 A/cm-width at $77 \mathrm{~K}$ ( $\mathrm{LN}_{2}$ boiling temperature) can now be made [7]. Since 2003, several pilot projects sponsored by the Department of Energy - Office Electricity and Energy Reliability (DOE-OE) have been successfully carried out at utility substations in the US, such as the Long Island superconductor transmission cable system, capable of transmitting $574 \mathrm{MW}$ of electric power.

\section{Figure 1 goes here}

Despite the tremendous progress made in this area, in order for superconducting cables to become reality, their cost/performance ratio needs further improvement. The critical objective today is to increase the amount of current the superconductor can carry without dissipation in a range of magnetic fields required by the specific application. This can be achieved by carefully playing on two different and related fronts-trying to push the dissipation-free current density $\left(\mathrm{J}_{\mathrm{c}}\right)$ as close as possible to the theoretical limit, and at the 
same time, increasing the thickness of the superconductive layer as much as possible without degrading performance. It is in this final challenge that the newest achievements in nanotechnology come into play as $J_{\mathrm{c}}$ enhancements in thick superconducting films can only be obtained by introducing and engineering extrinsic nano-sized defects within the film.

\section{Flux pinning by nanoscale defects}

The importance of nanoscale defects derives from the concepts of magnetic flux lines and flux pinning in the superconductor. The current carried by a type II superconducting film, such as a YBCO film, generates a magnetic field (self field) that penetrates the superconductor in the form of tubular structures called vortices or flux lines, each of them carrying one unit of magnetic field, the flux quantum $\phi_{\mathrm{o}}$. The current interacts with the magnetic flux lines generating a Lorenz-type force (equal to $\mathbf{J} \times \boldsymbol{\phi}_{\mathbf{0}}$ ) that pushes the vortices across the conductor [8-12]. Because the material within the vortex core is in the normal state, this motion generates dissipation and the superconductor ends up resembling a low resistance conductor. Fortunately, naturally occurring nano-sized defects and secondary phases provide spatial variations in the thermodynamic free energy that act like potential wells for the flux lines. A flux line has a lower free energy when it is positioned in the energy well of the defect than it does in the bulk matrix. This energy difference acts as a pinning force constraining the vortex to remain within the well [1316]. Figure 2 is a schematic that illustrates the interaction between flux lines and different types of defects in a superconducting film. As the current increases, so does the Lorenz 
force and when this exceeds the pinning force $\left(a t \mathrm{~J}=\mathrm{J}_{\mathrm{c}}\right.$ ) dissipation begins. Therefore, the higher the pinning force, the higher the current the superconductor can sustain without dissipation.

\section{Figure 2 goes here}

The picture gets more complicated in presence of an external magnetic field, which is the case for several power applications. In this case, as more flux lines are generated within the superconductor, the density of pinning sites, in addition to their strength, becomes important. The most effective pinning sites are defects whose size is close to the vortex's normal core diameter $2 \xi$ where $\xi$, the superconducting coherence length, is a few nanometers for the superconductors of interest. This is why artificially introduced nanostructures play a dramatic role in optimizing pinning without destroying an unnecessary volume fraction of the superconductor, thereby allowing for a significant increase in $\mathrm{J}_{\mathrm{c}}$.

\section{The grain boundary problem}

Although flux pinning has been the object of numerous studies for many years, efforts aimed at increasing flux pinning in coated conductors by means of extrinsically added defects and nanostructures started only around 2004. The reason for this late interest is that early efforts to optimize coated conductors performance had to face more pressing issues. One of the greatest quandaries was that, even after more ordinary materials issues, 
such as interdiffusion of chemical species between the different layers and non-epitaxial growth were addressed, early CC's still showed reduced $\mathrm{J}_{\mathrm{c}}$ 's as compared to the $\mathrm{J}_{\mathrm{c}}$ of YBCO films of the same thickness on single crystal substrates (e.g. $\mathrm{SrTiO}_{3}$ ). The reason for this discrepancy resided in the imperfect texture of the metal-based template, which showed grain boundaries as high as 10 degrees. From early studies of YBCO films on bicrystals, scientists had learned that grain boundaries of this magnitude dramatically suppress $J_{c}$ across the grain boundary region (intergrain $J_{c}$ ) [17]. Therefore, strategic research on CC's towards the end of the 90's focused mainly in studying $\mathrm{J}_{\mathrm{c}}$ across $\mathrm{YBCO}$ grain boundaries in the low angle regime $\left(2^{\circ}-10^{\circ}\right)$ to establish the critical angle at which the intergrain $J_{c}$ started departing from the $J_{c}$ within the grain (intragrain $J_{c}$ ), and in improving CC's texture. The grain boundary problem, and related role of weak links in YBCO, casted a doubt on the technological application of CC's for many years but was finally resolved for both major routes of fabricating CC's through the improvement of the superconducting film texture $[18,19]$. Today both techniques are able to produce long wires with a true in-plane texture full-width-half-maximum (FWHM) in the YBCO film of $\sim 4^{\circ}$. $\mathrm{J}_{\mathrm{c}}$ 's comparable to those obtained on single crystals are obtained for wires with such sharp textures. While FWHM's less than $4^{\circ}$ are routinely obtained in IBAD coated conductors, RABiTS conductors still show some margin for improving the in-plane texture in the YBCO film. However, comparable $J_{c}$ values in IBAD and RABiTS conductors suggest that grain boundary meandering during YBCO growth on RABiTS might reduce the current-suppressing grain boundary effect in these conductors [20]. Grain boundaries less than $2^{\circ}$ do not essentially affect the performance of YBCO films and actually might be beneficial since their widely spaced dislocation cores provide 
additional pinning in magnetic fields. As a result of the study of low angle grain boundaries, it was found that the intergrain $\mathrm{J}_{\mathrm{c}}(\mathrm{H})$ curve, although lower than the intragrain $\mathrm{J}_{\mathrm{c}}(\mathrm{H})$ curve always joined the latter at a certain field value (see Fig. 3), which increased as the grain boundary angle increased [21]. For fields larger than this crossover field the grain boundary can in principle sustain a higher current density than the grain itself. This finding implies that 1) since all applications of CC's involve some magnetic field, a substrate with a texture of $3^{\circ}-4^{\circ}$ is equivalent to a single crystal substrate in terms of current performance. In addition, because for fields larger than the crossover field, $\mathrm{J}_{\mathrm{c}}$ is limited by the intragrain component, further $\mathrm{J}_{\mathrm{c}}$ improvements in this regime can only be achieved by optimizing bulk flux pinning. Additional fixes on the grain boundaries, such as, for example, $\mathrm{Ca}$ doping [22] will not produce any observable benefit. Once the limitations imposed by the grain boundaries were reduced, the efforts turned to improving flux pinning by introducing atomic-scale and nano-scale defect structures in addition to those naturally occurring during YBCO processing.

\section{Figure 3 goes here}

It is worth noting that while the performance of YBCO films on single crystal was considered an upper limit in early CC's days, such performance lies well below the theoretical limit. The critical current density at which superconductivity viewed as pairing of electrons is destroyed in the absence of any extrinsic material issue is named the depairing current and is roughly 3-4 times higher than the best $J_{c}$ values obtained today on any YBCO film. Therefore, CC's with performances well above those of thin 
YBCO films on single crystal are in principle possible. The questions to be addressed at this point are 1) what kind of nanostructures will offer the best pinning properties, and 2) how can these nanostructures be implemented in various YBCO processing techniques.

\section{Anisotropic current properties}

Since the deposition of the first YBCO films on single crystals, many studies have been conducted to investigate the origin for the high $\mathrm{J}_{\mathrm{c}}$ of epitaxial films as compared to melt textured and single crystal YBCO samples. The discovery of a well defined type of defect that would dramatically increase flux pinning throughout the H,T phase space has however eluded scientists and the picture that gradually emerged was that of a multitude of point-like and extended defects that acted synergistically producing different outcomes in different regimes. The reason for this complex behavior is that the pinning force depends on many characteristics of a single defect (size, shape, composition) and is not additive for different types of defects. In addition, pinning is affected by vortex-vortex interactions, which become more prominent at higher magnetic fields (the vortices are more numerous and closer together), by thermal activation processes, which can induce depinning of vortices, and by the electronic mass anisotropy, which is due to the layered crystal structure. As a result, the objective is not to increase pinning in general but to target the enhancement in particular $\mathrm{H}, \mathrm{T}$ regions of interest and for specific orientations of the magnetic field relative to the sample. Often, the pinning enhancement might be very significant in some $\mathrm{H}, \mathrm{T}$ regions and completely negligible or even deleterious in others. For example, a highly crystalline YBCO film with very few but strong columnar 
defects along the $c$ axis might have a record high $\mathrm{J}_{\mathrm{c}}$ in self-field [23] because all the vortices produced by the small self magnetic field can be accommodated in deep narrow potential wells, which take away very little superconducting material from the nearly perfect surrounding matrix. However, once the vortices exceed the number of columnar defects, they cannot be effectively pinned and $\mathrm{J}_{\mathrm{c}}$ decreases so rapidly with field that the film performance becomes completely inadequate for any real application. .

As a consequence of the anisotropic crystal structure and the presence of many point-like random defects, $\mathrm{J}_{\mathrm{c}}$ in as grown $\mathrm{YBCO}$ films obtained by various techniques is always maximal when the magnetic field is directed parallel to the $a b$ planes, a situation which is not preferred in all power applications. Figure 4 shows a typical curve for $\mathrm{J}_{\mathrm{c}}$ as function of magnetic field orientation for an YBCO film grown by pulsed laser deposition (PLD) on $\mathrm{SrTiO}_{3}$. Superimposed to the data points is a curve that represents the contribution to $\mathrm{J}_{\mathrm{c}}$ from uncorrelated pinning, produced by randomly distributed localized defects. The random pinning contribution is obtained by fitting the $J_{c}(\theta)$ data to the scaling curve predicted for electronic mass anisotropy effects alone, according to the analysis of Civale et al. [24]. The difference in $\mathrm{J}_{\mathrm{c}}$ between the data and the random pinning component is due to correlated pinning provided by extended, parallel pinning centers, either linear or planar, which naturally arise during growth. These defects are usually oriented both along the $c$ axis (especially for PLD films) and parallel to the $a b$ planes. A final contribution to the peak at $\mathrm{H} / / a b$ is given by intrinsic pinning, also due to the layered crystal structure.

From the point of view of meeting power applications criteria, the goal would be to introduce artificial pinning sites that enhance $J_{c}$ for orientations close to $H / / c$ near to the level of $\mathrm{J}_{\mathrm{c}}$ at $\mathrm{H} / / a b$, giving rise to a more isotropic angular dependence. 


\section{Figure 4 goes here}

Another way to quantify desirable pinning effects is to analyze the $\mathrm{J}_{\mathrm{c}}(\mathrm{H})$ curve for the least favorable field orientation $(\mathrm{H} / / c)$. Typically, in YBCO with only naturally occurring defects, $\mathrm{J}_{\mathrm{c}}(\mathrm{H})$ shows a plateau at small fields of the order of $0.1 \mathrm{~T}$, where the defects outnumber the vortices (see Fig. 5). Generally, added pinning sites produce a small effect in this regime because there are already enough defects to pin all the vortices produced by the small field. Although some added defects (such as impurity nanophases) may produce stronger pinning forces than other naturally occurring defects, they are also made of non-superconducting material and necessarily reduce the superconducting areal section and often $T_{c}$ as well. At intermediate fields, $\mathrm{J}_{\mathrm{c}}$ decreases like $\mathrm{H}^{-\alpha}$ where $\alpha$ is an exponent ranging between 0.5 and 0.7 . However, in the case of strong pinning, such as that produced by nanoparticles/nanorods additions, the $\alpha$ exponent decreases, reflecting a smaller $\mathrm{J}_{\mathrm{c}}$ degradation with increasing magnetic field. This is the regime were the introduction of artificial pinning sites plays the largest effect. For fields larger that $2 \mathrm{~T} \mathrm{a}$ rapid decay of $J_{c}$ toward zero at the irreversibility field $\mathrm{H}_{\text {irr }}$ is observed because of depressed vortex line tension and strong thermal activation. Improved performance in this regime can also be observed with added pinning sites, as observed for strong linear defects with high areal density.

\section{Figure 5 goes here}




\section{Enhancing naturally occurring nanoscale defects}

It is well known from flux pinning studies that only defects that are to some degree extended or correlated along the $c$ direction can give rise to enhancement of $\mathrm{J}_{\mathrm{c}} \mathrm{when} \mathrm{H} / / \mathrm{c}$. Similar to earlier heavy ion irradiation studies on single crystals [25], the best performance results from columnar structures of non-superconducting material that accommodate the vortex core over the entire thickness of the film with a certain amount of splay $[26,27]$. On the other hand, randomly distributed localized defects (uncorrelated pinning centers) can produce very strong pinning, but do not give rise to a peak for $\mathrm{H} / / \mathrm{c}$ in the $J_{c}$ angular dependence at $77 \mathrm{~K}$. One of the first attempts of correlating $J_{c}$ performance for $\mathrm{H} / / c$ with the density of extended pinning centers along the $c$ axis was carried out in 1999 [28]. The defects targeted by this study were threading edge and screw dislocations normally occurring during the $\mathrm{YBCO}$ growth process, especially in PLD films with their columnar growth mode. Subsequently, another type of strong, naturally occurring, pinning defects, known as antiphase boundaries, were studied in detail using controlled vicinal substrate surfaces to tailor their density [29, 30]. Figure 6 shows a collection of various defects usually occurring in YBCO films as imaged by high resolution transmission electron microscopy (HRTEM).

\section{Figure 6 goes here}

One approach to optimize flux pinning is to try to tune deposition conditions and/or the substrate morphology in order to increase the most effective naturally occurring crystal defects. For example, by controlling the size of the YBCO growth islands and the mosaic 
spread within a single substrate grain, additional threading dislocations along the $\mathrm{c}$ axis can be introduced. However, the increase in defect density obtained through this route is modest and limited to low magnetic fields. Moreover, such an approach is highly dependent on the YBCO deposition method.

By decorating the substrate surface with chemically compatible nanoparticles one can try to disrupt the subsequent $\mathrm{YBCO}$ growth just enough to introduce low angle grain boundaries or dislocations of another nature that might tread to the film surface. This approach has been pursued by various research groups in the last few years using different materials deposited in the form of nanoparticles on the substrate (Ir, $\mathrm{Ag}, \mathrm{Y}_{2} \mathrm{O}_{3}$, $\mathrm{ZrO}_{2}, \mathrm{MgO}, \mathrm{BaZrO}_{3}$ ) or processing the oxide template so that it develops outgrowths of nanoscale size [31-36]. However, the pinning induced by the defects and strain fields stemming from the surface particles is generally of a random nature and does not provide the desirable enhancement of $\mathrm{J}_{\mathrm{c}}$ for $\mathrm{H} / /$ c. Figure 7 shows an example of surface decoration on a $\mathrm{SrTiO}_{3}(001)$ substrate by PLD deposited $\mathrm{ZrO}_{2}$ nanodots. The YBCO growth is disturbed by the presence of the nanodots, and antiphase boundaries terminating in stacking faults are produced in the growing film. In two cases $c$-axis correlated defects with consequent significant $\mathrm{J}_{\mathrm{c}}$ enhancement for $\mathrm{H} / / \mathrm{c}$ were reported using $\mathrm{Y}_{2} \mathrm{O}_{3}$ nanoparticles and nanometer sized $\mathrm{SrTiO}_{3}$ outgrowths $[34,37]$. Nevertheless, an understanding of the correlation between nanoparticles or outgrowths and the type of defects they generate is lacking and similar nanostructures on the surface of the substrate give rise to different pinning mechanisms within the superconductor. On the other hand, a recent result obtained on ex situ YBCO films that did not contain artificially introduced pinning sites shows that the relation between defects and resulting pinning landscape is 
still not well understood and $c$-axis correlated defects might not be the only route for enhanced performances [38]. In that study, crystal defects that were not unambiguously identified, gave rise to very strong pinning, yielding an almost isotropic $J_{c}(\theta)$ curve at magnetic fields as high as $3 \mathrm{~T}$.

\section{Figure 7 goes here}

\section{Artificial introduction of flux pinning nanostructures}

A different approach, that has produced overall better results, is to introduce nanosized particles of chemically compatible, non-superconducting material throughout the superconductor matrix. This can be accomplished by deposition from multiple sources, in the case of in situ methods, or by adding excess elements to the superconductor precursor, in the case of ex situ methods. In situ ablation from a superconductor pellet already containing the nanoparticle material in a disperse form is another option. Although the uncorrelated pinning from randomly distributed point-like defects is generally weak, randomly dispersed finite size particles can provide strong pinning with significant enhancements throughout the H,T phase space. As several research groups worked on this approach, several examples of impurity additions in the form of nanoparticles and/or nanodots are available. In the first attempts an YBCO nonsuperconducting phase $\mathrm{Y}_{2} \mathrm{BaCuO}_{5}$, also known as 211 was added by intermittently depositing YBCO layers and discontinuous 211 layers by PLD using two deposition targets $[39,40]$. This approach resulted in pancake-like 211 particles with an average size of $15 \mathrm{~nm}$ distributed in the YBCO film, which produced some enhancement in pinning 
along $c$ and much higher improvements for $\mathrm{H} / / a, b$. Correlated pinning along the $c$-axis was also observed when seemingly randomly distributed 10 - $n m$-wide $\mathrm{BaZrO}_{3}(\mathrm{BZO})$ particles were inserted in the YBCO film by ablating from a PLD target synthesized using $\mathrm{Ba}$ excess and added $\mathrm{Zr}$ [41]. In the last study the observed correlated pinning along the $c$-axis was related to misfit dislocations between the nanoparticles and the superconducting matrix, which aligned along the $c$ direction, with an areal density of 400

$\mu \mathrm{m}^{-2}$ compared to $80 \mu \mathrm{m}^{-2}$ for undoped YBCO films. Figure 8 shows high resolution Zcontrast scanning transmission electron microscope (STEM) images of a YBCO film with incorporated $\mathrm{Y}_{2} \mathrm{O}_{3}$ nanoparticles obtained with the multilayer approach (depositing from a $\mathrm{YBCO}$ and a $\mathrm{Y}_{2} \mathrm{O}_{3}$ target alternately) [42]. In this case, the $\mathrm{Y}_{2} \mathrm{O}_{3}$ nanodots ( 3 to 10 $\mathrm{nm}$ in size) grew epitaxially aligned with the $\mathrm{YBCO}$ in a coherent fashion, without obvious signs of lattice distortions, stacking faults or strain fields. Therefore, the observed pinning enhancement can be assumed to derive from the nanodots themselves and not from defects induced by the nanodots. The films in this study showed strong flux pinning characterized by a better $J_{\mathrm{c}}$ retention in magnetic field compared to pure YBCO films, as indicated by a lower value $(0.3$ vs. 0.5$)$ for the $\alpha$ exponent. However, the dependence of $J_{c}$ on the orientation of the magnetic field was typical of random pinning and showed no correlation or preferential direction for the pinning.

\section{Figure 8 goes here}

Nanoparticle addition has the great advantage of being applicable to all YBCO deposition methods, including solution-based ex situ processes. For example, nanocomposite YBCO 
films containing randomly oriented, non-coherent BZO nanoparticles in their matrix were recently processed from complex metal-organic solutions [43]. These films showed strong isotropic flux pinning generated by stacking faults and other lattice defects emanating in the YBCO from the embedded nanoparticles.

\section{Self-assembled nanostructures}

When BZO nanoparticles are epitaxially oriented within the YBCO matrix a significant strain field is expected in the growing film due to the large mismatch between the two lattices, which is about $9 \%$ when $\mathrm{BZO}(001) / / \mathrm{YBCO}(001)$ and $\mathrm{BZO}(100) / / \mathrm{YBCO}(100)$. Such strain, if properly tuned, can induce a remarkable self-assembly of the nanoparticles resulting in the growth of almost continuous and uniform $\mathrm{BZO}$ nanorods in the $\mathrm{YBCO}$ matrix. The mechanism responsible for self-assembly has been explored for semiconducting heteroepitaxial structures and is quite complex. Tersoff, et al. [44, 45] have demonstrated that in multilayer structures with large mismatch, buried dots can influence the nucleation in subsequent layers, leading to self-organization of a more ordered and uniform array. Formation of an island on the surface is most favorable where the strain at the surface reduces the mismatch between the surface and the island. Because the nucleation rate is an exponential function of the nucleation barrier, which in turn depends sensitively upon the strain, islands nucleate wherever this strain gives a local minimum in the misfit. Strain mediated self-assembly of nanodots is therefore more prominent for large mismatched heteroepitaxial systems. Analogous to semiconductor heterostructures, a multilayer approach in which continuous YBCO layers and BZO 
nanodots are deposited sequentially is expected to produce a near ideal regular array of linear defects embedded within the HTS film matrix, when the layers thickness is properly controlled.

Goyal [46, 47] and MacManus-Driscoll [48] were able to produce such an ordered array of BZO nanodots in YBCO by simply performing laser ablation from a single target comprising a mixture of $\mathrm{YBCO}$ powder and BZO nanoparticles. During simultaneous deposition of YBCO and $\mathrm{BZO}$, the two phases were found to separate in the growing film and interact through the strain field similar to consecutively deposited phases. Goyal [49] and Varanasi [50] were able to produce similar nanostructures using different oxides in addition to $\mathrm{BZO}\left(\mathrm{CaZrO}_{3}, \mathrm{YSZ}, \mathrm{MgO}, \mathrm{Ba}_{\mathrm{x}} \mathrm{Sr}_{1-\mathrm{x}} \mathrm{TiO}_{3}, \mathrm{BaSnO}_{3}\right)$ and therefore demonstrate the general validity of this approach. In addition to the strain field that develops around the nanoscale second phases embedded in the matrix, reduction of phase boundary energy also plays an important role for simultaneous deposition of $\mathrm{YBCO}$ and $\mathrm{BZO}$, causing the morphology of the second phases to resemble continuous nanorods instead of a stack of nanodots. The nanorods can in some cases extend for the entire film thickness or consist of stacks of shorter nanorods. Theoretical formulations based on energetic and kinetic arguments specify an optimal range of $4-12 \%$ for the lattice mismatch of the embedded phase with respect to the REBCO matrix.

Figure 9 shows a HRTEM image obtained from an $\mathrm{YBCO} / \mathrm{BZO}$ composite films deposited from a YBCO target doped with 2 vol. \% BZO nanoparticles. The BZO columns produced by stacking of nanodots and continuous nanorods are clearly visible in the TEM cross-section. The contrast of the BZO rods is generally enhanced in the TEM images by tilting the sample along the $\mathbf{g}=001$ vector of YBCO. The nanocolumns extend 
throughout the thickness of the film, have a diameter of $2-3 \mathrm{~nm}$, and are aligned along the $c$ axis of YBCO.

\section{Figure 9 goes here}

Figure 10 shows a plan view TEM image in low magnification with the electron beam directed parallel to the $\mathrm{YBCO} c$ axis. It is interesting to note that the areal distribution of nanocolumns as shown in this image is not random and the spacing between nearest neighboring columns is fairly uniform, suggesting an interaction-mediated selforganization in the basal plane of the film. The degree of order in the areal distribution of columns can be probed by plotting the autocorrelation function for a bidimensional plot of the nanocolumn positions in Fig 10a. The autocorrelation function of an image I is defined as the inverse Fourier transform of $F(I) \times F^{*}(I)$, where $F(I)$ indicates the Fourier transform of the Image and $\mathrm{F}^{*}(\mathrm{I})$ is the complex conjugate. As the autocorrelation determines the self-similarity of an image, the result always shows a peak in the center (each image point is correlated to itself) and an additional structure that ranges from an amorphous background, for a random distributions of dots, to an ordered peak array, for an ordered lattice of dots. As shown in Fig. 10b, several distinct peaks are observed for the BZO columns distribution around the central maximum and their symmetry resembles that of a square lattice with constant $a \cong 15 \mathrm{~nm}$. From this average spacing we can estimate a maximum pinning force scenario for an applied magnetic field $\mathrm{B}_{\phi}=\phi_{\mathrm{o}} / a^{2}$ of $8-10 \mathrm{~T}$, known as the matching field, corresponding to the ideal situation of each nanocolumn accommodating one flux line. According to this value, significant $\mathrm{J}_{\mathrm{c}}$ 
enhancement is expected in these films for all field regimes that relevant for certain power applications.

\section{Figure 10 goes here}

The high-resolution Z-contrast STEM image of Fig. 11 is a plan view of a single BZO nanorod. Different atomic columns are distinguishable thanks to a dependence of the image intensity on the atomic number. In the $\mathrm{YBCO}$ matrix the $\mathrm{Ba} / \mathrm{Y}$ columns appear brighter than the $\mathrm{Cu} / \mathrm{O}$ columns and within the $\mathrm{BZO}, \mathrm{BaO}$ columns appear brighter than $\mathrm{ZrO}$. The image shows that the BZO is epitaxially oriented and coherent with the YBCO matrix, and misfit dislocations form to accommodate mismatch at the corners of the BZO nanorod. Since the nanorods are aligned along the $\mathrm{YBCO} c$ direction, the strain from the misfit dislocations is also aligned and extended along this direction. Both the arrays of dislocations and the nanorods themselves are therefore expected to form ideal flux pinning columns, similar to damage tracks by heavy ion irradiation [51, 52].

\section{Figure 11 goes here}

The pinning enhancement is evident in Fig.12, which compares $J_{c}$ dependencies on field magnitude and orientation for an undoped YBCO film on RABiTS and a BZO-doped YBCO film on the same substrate. Both films have a thickness of $0.2 \mu \mathrm{m}$. In particular, we notice that the correlated $c$ axis peak in the angular dependence of $J_{c}$ is very pronounced for the YBCO film containing the BZO nanodots, indicative of strong 
pinning defects along the $c$ axis. Although the $\mathrm{J}_{\mathrm{c}}$ 's shown in Fig. 12 are large, for practical application of HTS it is also essential to increase the overall current carrying capacity of the coated conductor. The figure of performance of a coated conductor is not the $\mathrm{J}_{\mathrm{c}}$ of the $\mathrm{YBCO}$ film but the engineering current density $\mathrm{J}_{\mathrm{E}}$ of the whole conductor, which is the total current carried by the superconductor, $\mathrm{I}_{\mathrm{c}}$, divided by the total cross section of the conductor. Because the thickness of the superconducting film is only a small fraction $(2-3 \%)$ of the total conductor thickness, including the substrate and the stabilizing metal overlayer (see Fig. 1), YBCO films with a thickness of a few microns, and therefore larger $\mathrm{I}_{\mathrm{c}}$ 's are in fact preferred, provided their $\mathrm{J}_{\mathrm{c}}$ is similar to that of $0.2-\mu m$ thick films. Unfortunately, gradual deterioration of the critical current density occurs with increasing superconductor thickness, and in some cases, a dead layer, which provides no contribution to the current carrying ability, forms after a critical thickness of $\sim 1.5 \mu \mathrm{m}$.

\section{Figure 12 goes here}

This effect has been attributed both to extrinsic material issues, such as roughening of the film/vapor interface, coarsening of precipitates, secondary outgrowths, etc., and the intrinsic healing with thickness of treading misfit dislocations originating at the substrate interface. A first solution to this problem was found by fabricating multi-layered films with alternating YBCO layers less than $1.5 \mu m$ thick and intervening $\mathrm{CeO}_{2}$ interlayers [53]. More recently, several group were able to overcome the extrinsic issues involved with the $\mathrm{J}_{\mathrm{c}}$ decay in thick films by optimizing PLD YBCO deposition conditions, and/or using a mixture of $\mathrm{Y}$ and $\mathrm{Eu}$ instead of pure $\mathrm{Y}$ to obtain a smoother and denser film 
microstructure [54]. Goyal et al. have recently demonstrated record-high critical current values by incorporating 3D self-assembled BZO nanodots, like the ones shown above, in films as thick as $4 \mu \mathrm{m}$. The achievement of 3D self-assembly throughout such large thickness was obtained by careful control of the PLD target concentration, homogeneity, and surface roughness, and by optimization of deposition repetition rate and substrate temperature [55]. Figure 13 shows cross-section TEM images of the $4 \mu \mathrm{m}$-thick film, illustrating the BZO nanorod structure in the film. It appears that the columns of selfassembled BZO nanodots are not perfectly aligned along the $c$-axis of $\mathrm{YBCO}$ at the high deposition rate used to grow the film. The columns show a range of angles with respect to the $c$-axis of YBCO and resemble a 'splayed' defect microstructure. Such a 'splayed' microstructure appears to be more favorable for flux-pinning at all field orientations [27]. The performance of this $4 \mu m$-thick film on a short section of CC template $\left(I_{\mathrm{c}}\right.$ of $353 \mathrm{~A}$ $\mathrm{cm}^{-1}$ at $\mathrm{T}=65 \mathrm{~K}, \mathrm{H}=3 \mathrm{~T}$ ) is higher than record values previously reported and exceed minimum requirements for transmission cables, electric ship propulsion systems, largescale motors, and generators. This result is even more impressive when considering that the BZO nanodots technology was successfully transferred from ORNL to SuperPower, one of the leading US Company in $2 \mathrm{G}$ coated conductors manufacture. Interestingly, the self-assembly of BZO dots is not confined to the PLD process but also occurs, with proper processing tuning, during YBCO growth by MOCVD, a highly scalable technique used by Superpower to produce kilometer long superconducting cables [56].

\section{Figure 13 goes here}

Effect of local strain fields in nanocomposite films 
In composite superconducting films, the incorporated nanocolumns give rise to local strain modulations, which have important effects not only on pinning but also on oxygen stoichiometry and $T_{\mathrm{c}}$. Differently from (and in addition to) the macroscopic, unidirectional strain that arises in YBCO films due to epitaxial growth on a substrate with slightly different lattice parameter, the array of vertical interfaces between BZO nanocolumns and YBCO matrix produces a much more localized, non-monotone strain field, which varies on the nanoscale and produce measureable broadening of XRD peaks. XRD analysis shows that such a local strain increases with increasing BZO concentration and thus nanocolumns density, as shown by the square symbols in Fig. 14b for both PLDand MOCVD- deposited films [55, 57, 58]. Finite element strain distribution calculations for the YBCO-BZO system (Fig. 14c) deviate significantly from the XRD measurements, as shown by the triangular symbols in Fig. 14b. Such a discrepancy originates from misfit dislocations present at the vertical $\mathrm{YBCO} / \mathrm{BZO}$ interfaces, which render these interfaces non-coherent. Modeling dislocations within the finite element calculation does indeed produce microstrain estimates closer to the experimental values, as shown by circles in Fig $14 \mathrm{~b}$ for the less computational intensive case of diluted nanocolumns, for which the ratio between nanocolumn length, $d$, and distance between nanocolumns, $L ; d / L=1$. The experimental films considered in this study fall in the more concentrated limit $d / L \sim 20$.

\section{Figure 14 goes here}

In addition to an increase in local strain, an increasing BZO concentration above $\sim 4$ vol $\%$ also produces a significant drop in $\mathrm{T}_{\mathrm{c}}$ with a linear trend of $\sim 0.4 \mathrm{~K} /$ vol $\%$. 
Understanding the cause of such unexpected drop in $T_{c}$ and reconciling microstrain measurements with theoretical predictions was possible using aberration-corrected Zcontrast STEM coupled with EELS, which allows lattice displacement and chemical concentration analysis at the nanoscale [59]. Figure 15 shows a single BZO nanorod sectioned for TEM using the focus ion beam (FIB) technique. The nanorod is embedded in a REBCO film (RE is a mixture of $\mathrm{Y}$ and $\mathrm{Gd}$ ) grown by MOCVD and having a BZO volume fraction of $4.1 \%$. The Z-STEM image shows that tilt and misfit between the relative lattices of rod and matrix is accommodated by tilt dislocations with Burgers vector $1 / 2(100)$, as indicated by circles, and misfit dislocations with Burgers vector 1/2(101), which are active in relieving misfit strain (indicated by arrows). The average spacing between dislocations is $\sim 3$ YBCO unit cells $(3.5 \mathrm{~nm}$ ). A similar average spacing $D$ can be calculated using the equation $D=b_{\mathrm{z}} /\left(f_{\mathrm{o}}-\varepsilon_{\mathrm{o}}\right)$, where $f_{\mathrm{o}}$ defines the original misfit between lattices, $\varepsilon_{\mathrm{o}}$ is the residual misfit strain, and $b_{\mathrm{z}}$ is the Burgers vector component along the interface plane. Choosing $\varepsilon_{0}=1.9 \%$, as suggested by Fig. 14 comparing theoretical and measured microstrain ${ }^{1}$ gives $D=2.7$ YBCO unit cells, showing excellent agreement between STEM and XRD. The local strain can be quantified directly through the Z-STEM images by measuring the variation of the REBCO $c$ axis parameter. The variation in $c$ axis spacing shown in Fig. $15 \mathrm{~b}$ is derived by finding the center of mass for the intensity distribution of each $\mathrm{Ba}$ and $\mathrm{Y} / \mathrm{Gd}$ column in the desired region (dashed box in the figure) and then averaging the calculated $c$ spacings in the direction parallel to the

\footnotetext{
${ }^{1}$ Fig $14 \mathrm{~b}$ shows that the experimental slope is reduced to nearly $24 \%$ of the simulation for the case $\mathrm{d} / \mathrm{L}=$ 20 and coherent interface, indicating that $76 \%$ of the original misfit has been relieved and the effective residual misfit strain is $\sim 24 \% \times 8 \%=1.9 \%$. This reduction is caused by misfit dislocations that form at the $\mathrm{YBCO} /$ $\mathrm{BZO}$ interface, giving rise to a semicoherent $\mathrm{REBCO} /$ BZO phase boundary.
} 
interface. The quantity shown in the figure is the difference between this average and the bulk lattice spacing $c_{0}$ well within the matrix, $\left(c-c_{0}\right) / c_{0}$. The data are compared in the same figure with the theoretical prediction obtained by analytically solving the anisotropic elastic boundary value problem of the bimaterial interface dislocations [60].

\section{Fig. 16 goes here}

The theoretical trend is similar to the data extracted by Z-STEM with some difference in the location and magnitude of the peak, which can be attributed to an additional mechanism for strain relief unaccounted for by the theoretical model: the presence of oxygen vacancies. The existence of a relationship between film stress and oxygen nonstoichiometry has been reported in the literature for perovskites at interfaces [61, 62] and grain boundaries [63]. In REBCO/BZO nanocomposite films, the local $\mathrm{O}$ stoichiometry was investigated by electron energy loss (EELS) elemental maps of film regions incorporating the rod, as shown in Fig 16. The simultaneously acquired, Zsensitive annular dark field (ADF) signal shows a bright contrast in the REBCO region immediately adjacent to the BZO rod, which is indicative of lattice distortions. The region showing the brightest shade in the ADF map appears as darkest in the O-K map. That is, the $\mathrm{O}$ signal shows a minimum in the REBCO region immediately surrounding the rod, which is highly strained according to the discussion above. In contrast, the $\mathrm{Ba}$ and $\mathrm{Cu}$ maps show maximum and minimum values, respectively, within the $\mathrm{BZO}$ rod and exhibit a constant contrast level everywhere else in the surrounding REBCO matrix. Since the heavier atoms show a constant concentration outside the BZO rod, the dark 
contrast in the $\mathrm{O}$ map cannot be caused by electron beam dechanneling and is correlated to lattice strain. Local probing of the $\mathrm{Cu}$ valence using the $\mathrm{Cu}-\mathrm{L}_{2,3}$ edge is another way to assess oxygen deficiency. As shown by the ratio of the $\mathrm{L}_{3}$ and $\mathrm{L}_{2}$ lines, the $\mathrm{Cu}$ valence is lower in region 1, adjacent to the $\mathrm{BZO}$ rod than in region 2, within the REBCO matrix, indicating lower $\mathrm{O}$ coordination at the interface with the nanorod. The same behavior for the $\mathrm{Cu}-\mathrm{L}_{2,3}$ edges is observed in all of the spectrum images acquired, indicating that the oxygen deficiency is present everywhere within the strained region surrounding the nanorods and is not localized in certain points. A picture then emerges in which the strain surrounding the nanorods hinders proper REBCO oxygenation, and the resulting substoichiometry reduces somewhat the strain at the phase boundary, partially explaining the discrepancy with analytical calculations (Figure 15b). Oxygen deficiency readily suppresses $T_{c}$ in REBCO and the observed distribution of oxygen depleted regions explains the reduced $T_{c}$ in REBCO films with self-assembled BZO nanorods for concentrations of $4 \mathrm{vol} \%$ and above. The diameter of the BZO rods is $6-8 \mathrm{~nm}$ and the average spacing between BZO rods is $\sim 20 \mathrm{~nm}$ for a BZO concentration of 4 vol $\%$. The observed spatial extent of oxygen depletion is $3-4$ times the BZO rod diameter; therefore an overlap of oxygen-depleted REBCO regions surrounding BZO nanocolumns is expected to occur near or above this concentration, leading to the disruption of current percolation through the high $T_{c}$ phase and to a lower measured $T_{c}$. On the other hand, nanostrain modulations are highly effective in pinning vortices, and, in the case of randomly oriented BZO nanoparticles, the resulting pinning force is found to be isotropic [64], leading to an almost constant $\mathrm{J}_{\mathrm{c}}$ as a function of magnetic field orientation, which is highly desirable for applications involving superconducting coils. Dislocations and strain 
at REBCO grain boundaries also produces oxygen depletion, which, however, can be mitigated by impurity additions (e.g.,Ca) $[63,65]$. Therefore, doping might be effective in locally raising $T_{c}$ while, at the same time, maintaining the positive pinning effect of nanostrain, hence further improving the critical current in nanocomposite superconducting films.

\section{Fig. 17 goes here}

\section{Control of epitaxy-enabling atomic sulfur superstructure}

In addition to the nanostructures added to the superconductor to enhance its performance, the successful processing of long lengths of $2 \mathrm{G}$ HTS wires is based on the employment of several other nanotechnologies. A great example of nanotechnology use in coated conductors is given by the atomic surface treatment of the Ni-W surface prior to the deposition of oxide buffer and superconductor layers in RABiTS coated conductors. Before 2001, it seemed natural to assume that a deposited oxide layer nucleated on a clean and pure Ni or Ni-W surface. Before deposition, the native metal oxide was removed by an in situ reducing heat treatment, and unwanted re-oxidation of the $\mathrm{Ni}$ surface was prevented by choosing an oxide layer thermodynamically more stable than $\mathrm{NiO}$ (e.g., $\mathrm{CeO}_{2}$ or $\mathrm{Y}_{2} \mathrm{O}_{3}$ ) and by depositing it in reducing conditions for $\mathrm{Ni}$. At this point, the lattice match between metal and oxide buffer layer was supposed to be the main factor influencing epitaxial growth. This picture, however, ultimately proved to be inadequate, particularly after the introduction of continuous reel-to-reel processes for fabricating kilometers-long buffered tapes. In fact, irreproducibilities in the oxide layer 
texture and unwanted crystal orientations threatened to stop further development of coated conductors in the early stage of industrial scale-up. Careful structural and chemical investigations subsequently revealed the existence of a $c(2 \times 2)$ sulfur superstructure on the textured $\mathrm{Ni}$ surface that formed after diffusion and surface segregation of sulfur contained in the metal bulk at a level less than $30 \mathrm{wt}$ ppm [66]. It was found that a fully developed atomic sulfur superstructure on the $\mathrm{Ni}$ surface, corresponding to half monolayer of S atoms, enabled the epitaxial nucleation of the oxide buffer layer and consequently the right crystal orientation for the superconductor. In cases where the bulk S contents of particular batches of Ni or Ni-W were much lower than $30 \mathrm{wt} \mathrm{ppm}$, and therefore inadequate to spontaneously produce the surface superstructure, the buffer layer deposition process produced films with degraded texture or mixed crystal orientations. However, annealing the Ni-W substrate at $800{ }^{\circ} \mathrm{C}$ in the presence of a small amount of $\mathrm{H}_{2} \mathrm{~S}$ was sufficient to produce a stable $c(2 \times 2)$-S with the desired coverage of 0.5 ML [67]. This simple step allowed creating a complete S template independently of the coverage obtained through segregation. Implementing this step in a continuous RABiTS fabrication process provided a solution to the irreproducibility issues and enabled subsequent stages of development. The role played by the $\mathrm{S}$ superstructure can be partially explained on the basis of structural and chemical considerations. The $\mathrm{S}$ layer behaves like a template that matches and mimics the arrangement of the oxygen atoms of particular (001) sublattice planes of $\mathrm{YSZ}, \mathrm{CeO}_{2}$, or STO. Sulfur belongs to the VI group and is chemically very similar to oxygen, often exhibiting the same electronic valence. Therefore, it is plausible that during the oxide buffer layer deposition the cations easily bond to the $\mathrm{S}$ atoms already present on the 
substrate surface, giving rise to the desired (001) epitaxial growth of the film, which otherwise would not take place. Such structural argument for the effect of the S template on buffer layer nucleation is supported by the observation that other oxides with crystal structures very similar to perovskites and fluorites, like $\mathrm{LaMnO}_{3}$, and $\mathrm{Gd}_{2} \mathrm{O}_{3}$ or $\mathrm{Y}_{2} \mathrm{O}_{3}$ also grow epitaxially on (100) $\mathrm{Ni}$ (or Ni-W) only in presence of an intervening $c(2 \times 2)$-S superstructure. Figure 14 illustrates the above proposed model of epitaxial growth on a Sterminated (001) Ni surface for $\mathrm{SrTiO}_{3}$ and $\mathrm{CeO}_{2}$. Today, $\mathrm{Y}_{2} \mathrm{O}_{3}$ is used instead of $\mathrm{CeO}_{2}$ or $\mathrm{SrTiO}_{3}$ as first oxide buffer layer for its improved structural and diffusion properties. However, the $\mathrm{Y}_{2} \mathrm{O}_{3}$ crystal structure is very similar to that of $\mathrm{CeO}_{2}$ and the model applies to $\mathrm{Y}_{2} \mathrm{O}_{3}$ as well.

\section{Figure 17 goes here}

\section{Acknowledgments}

The authors would like to thank Prof. L. Civale, Dr. D. Christen, and Dr. M. Varela for providing some of the figures. Research supported by the US Department of Energy, Office of Electricity Delivery and Energy Reliability and by the U.S. Department of Energy, Basic Energy Sciences, Materials Sciences and Engineering Division. The Research was performed in part at the Shared Research Equipment User Facility, a DOEBES user facility. 


\section{References}

[1] A. Goyal, D. P. Norton, J. D. Budai, M. Paranthaman, E. D. Specht, D. M.

Kroeger, D. K. Christen, Q. He, B. Saffian, F. A. List, D. F. Lee, P. M. Martin, C.

E. Klabunde,E. Hatfield, and V. K. Sikka, High critical current density

superconducting tapes by epitaxial deposition of $\mathrm{YBa}_{2} \mathrm{Cu}_{3} \mathrm{O}_{x}$ thick films on

biaxially textured metals. Appl. Phys. Lett. 69, 1795-1797 (1996).

[2] A. Goyal, M. Paranthaman, and U. Schoop, The RABiTS approach: Using rollingassisted biaxially textured substrates for high-performance YBCO

superconductors. MRS Bulletin 29, 552-561 (2004).

[3] A. Goyal, Epitaxial superconductors on rolling-assisted-biaxially-texturedsubstrates (RABiTS). Chapter in book "Second Generation HTS Conductor", edited by A. Goyal (Kluwer, Norwell, MA, 2005), pages 29-46.

[4] Y. Iijima, N. Tanabe, Y. Ikeno, and O. Kohno, Biaxially aligned $\mathrm{YBa}_{2} \mathrm{Cu}_{3} \mathrm{O}_{x}$ thin film tapes. Physica C 185, 1959-1960 (1991).

[5] X. D. Wu, S. R. Foltyn, P. N. Arendt, J. Townsend, C. Adams, P. Tiwari, J. Y. Coulter, D. E. Peterson, High current $\mathrm{YBa}_{2} \mathrm{Cu}_{3} \mathrm{O}_{x}$ thick films on flexible nickel substrates with textured buffer layers. Appl. Phys. Lett. 65, 1961-1963 (1994).

[6] P. Arendt, IBAD Template for HTS Coated Conductors. Chapter in book "Second Generation HTS Conductors”, edited by A. Goyal (Kluwer, Norwell, MA, 2005), pages $1-28$.

[7] See 2007 and 2008 presentations for DOE Annual Peer Review on Superconductivity available at http://www.energetics.com/supercon08/agenda.html. For further info, see also http://apps1.eere.energy.gov/news/news_detail.cfm/news_id=11609 
[8] P.J. Lee, Engineering Superconductivity (John Wiley and sons, Inc., New York, 2001).

[9] A. M. Campbell, and J. E. Evetts, Flux vortices and transport current in type-II superconductors. Adv. Phys. 21, 194-428 (1972).

[10] A. I. Larkin, and Yu. N.Ovchinnikov, Pinning in Type II superconductors. J. Low. Temp. Phys. 34, 409-428 (1979).

[11] G. Blatter, M. V. Feigel'man, V. B. Geshkenbein, A. I. Larkin, and V. M. Vinokur, Vortices in high-temperature superconductors. Rev. Mod. Phys. 66, 1125-1388 (1994).

[12] M. Tinkham, Introduction to Superconductivity (McGraw Hill, New York, 1975).

[13] D. R. Nelson, Points, lines and planes: vortex pinning in high-temperature superconductors. Physica C 263, 12-16 (1996).

[14] D. Agassi and J.R. Cullen, New vortex state in the presence of a long Josephson junction. Physica C 316, 1-12 (1999).

[15] D. Agassi, D. K. Christen, and S.J. Pennycook, Flux pinning and critical currents at low-angle grain boundaries in high-temperature superconductors. Appl. Phys. Lett. 81, 2803-2805 (2002).

[16] R. Kumar, S.K. Malik, S.P. Pai, R. Pinto, and D. Kumar, Self-field-induced flux creep in $\mathrm{YBa}_{2} \mathrm{Cu}_{3} \mathrm{O}_{7-y}$ thin films. Phys. Rev. B 46, 5766-5768 (1992).

[17] D. Dimos, P. Chaudhari, and J. Mannhart, Superconducting transport properties of grain boundaries in $\mathrm{YBa}_{2} \mathrm{Cu}_{3} \mathrm{O}_{7}$ bicrystals. Phys. Rev. B 41, 4038-4049 (1990). 
[18] S. R. Foltyn, P. N. Arendt, Q. X. Jia, H. Wang, J. L. MacManus-Driscoll, S. Kreiskott, R. F. DePaula, L. Stan, J. R. Groves, and P. C. Dowden, Strongly coupled critical current density values achieved in $\mathrm{YBa}_{2} \mathrm{Cu}_{3} \mathrm{O}_{7-\delta}$ coated conductors with near-single-crystal texture. Appl. Phys. Lett. 82, 4519-4521 (2003).

[19] A. Goyal, N. Rutter, C. Cantoni, and D.F. Lee, Long-range current flow and percolation in RABiTS-type conductors and the relative importance of out-ofplane and in-plane misorientations in determining $J(c)$. Physica $\mathrm{C}-$ Superconductivity and its Applications 426, 1083-1090.

[20] D. M. Feldmann, T. G. Holesinger, C. Cantoni, R. Feenstra, N. A. Nelson, D. C. Larbalestier, D. T. Verebelyi, X. Li and M. Rupich, Grain orientations and grain boundary networks of $\mathrm{YBa}_{2} \mathrm{Cu}_{3} \mathrm{O}_{7-d}$ films deposited by metalorganic and pulsed laser deposition on biaxially textured Ni-W substrates" Journal of Materials Research 21, 923 (2006).

[21] D. T. Verebelyi, D. K. Christen, R. Feenstra, C. Cantoni, A. Goyal, D. F. Lee, M. Paranthaman, P. N. Arendt, R. F. DePaula, and J. R. Groves, and C. Prouteau, Low angle grain boundary transport in $\mathrm{YBa}_{2} \mathrm{Cu}_{3} \mathrm{O}_{7-\delta}$ coated conductors. Appl. Phys. Lett 76, 1756-1758 (2000).

[22] A. Schmehl, B. Goetz, R.R. Schulz, C.W. Schneider, H. Bielefeldt, H. Hilgenkamp, J. Mannhart, Doping-induced enhancement of the critical currents of grain boundaries in $\mathrm{YBa}_{2} \mathrm{Cu}_{3} \mathrm{O}_{7-\delta}$. Europhysics Letters 47, 110-115 (1999). 
[23] J. Z. Wu, R. L. S. Emergo, X. Wang, G. Xu, T. J. Haugan, and P. N. Barnes, Strong nanopore pinning enhances $J_{c}$ in $\mathrm{YBa}_{2} \mathrm{Cu}_{3} \mathrm{O}_{7-\delta}$ films. Appl. Phys. Lett. 93, 062506-1 - 062506-3 (2008).

[24] B. Maiorov and L. Civale, Identification of vortex pinning centers and regimes in coated conductors. Chapter in book "Flux pinning and AC loss studies on YBCO coated conductors", edited by M. P. Paranthaman and V. Selvamanickam (Nova Science Publishers, Inc. New York, 2007) pages 35-58.

[25] L. Civale A. D. Marwick, T. K. Worthington, M. A. Kirk, J. R. Thompson, L. Krusin-Elbaum, Y. Sun, J. R. Clem, and F. Holtzberg, Vortex confinement by columnar defects in $\mathrm{YBa}_{2} \mathrm{Cu}_{3} \mathrm{O}_{7}$ crystals: Enhanced pinning at high fields and temperatures. Phys. Rev. Lett. 67, 648-651 (1991).

[26] T. Hwa, P. Le Doussal, D. R. Nelson, and V. M. Vinokur, Flux pinning and forced vortex entanglement by splayed columnar defects. Phys. Rev. Lett. 71, 3545-3548 (1993).

[27] L. Civale, L. Krusin-Elbaum, J. R. Thompson, R. Weeler, A. D. Marwick, M. A. Kirk, Y. R. Sun, F. Holtzberg, and C. Feild, Reducing vortex motion in $\mathrm{YBa}_{2} \mathrm{Cu}_{3} \mathrm{O}_{7}$ crystals with splay in columnar defects. Phys. Rev. B 50,4102-4105 (1994).

[28] B. Dam, J. M. Huijbregtse, F. C. Klaassen, R. C. F. van der Geest, G. Doornbos, J. H. Rector, A. M. Testa, S. Freisem, J. C. Martinezk, B. Staüble-Pümpin, and R. Griessen, Origin of high critical currents in $\mathrm{YBa}_{2} \mathrm{Cu}_{3} \mathrm{O}_{7-\delta}$ superconducting thin films. Nature 399, 439-442 (1999). 
[29] T. Haage, J. Zegenhagen, J. Q. Li, H.-U. Habermeier, M. Cardona, Ch. Jooss, R. Warthmann, A. Forkl, and H. Kronmüller, Transport properties and flux pinning by self-organization in $\mathrm{YBa}_{2} \mathrm{Cu}_{3} \mathrm{O}_{7-\delta}$ films on vicinal $\mathrm{SrTiO}_{3}(001)$. Phys. Rev. B 56, 8404-8418 (1997).

[30] C. Cantoni, D. T. Verebelyi, E. D. Specht, J. Budai, and D. K. Christen, Anisotropic nonmonotonic behavior of the superconducting critical current in thin $\mathrm{YBa}_{2} \mathrm{Cu}_{3} \mathrm{O}_{7-\delta}$ films on vicinal $\mathrm{SrTiO}_{3}$ surfaces. Phys. Rev. B 71, 054509-1 054509-9 (2005).

[31] A. Crisan, S. Fujiwara, J. C. Nie, A. Sundaresan, and H. Ihara, Sputtered nanodots: a costless method for inducing effective pinning centers in superconducting films. Appl. Phys. Lett. 79, 4547-4549 (2001).

[32] T. Aytug, M. Paranthaman, K. J. Leonard, S. Kang, P. M. Martin, L. Heatherly, A. Goyal, A. O. Ijaduola, J. R. Thompson, D. K Christen, R. Meng, I. Rusakova, and C. W. Chu, Analysis of flux pinning in $\mathrm{YBa}_{2} \mathrm{Cu}_{3} \mathrm{O}_{7-\delta}$ films by nanoparticlemodified substrate surfaces. Phys. Rev. B. 74, 184505-1 - 184505-8 (2006).

[33] T. Aytug, M. Paranthaman, A. A. Gapud, S. Kang, H. M. Christen, K. J. Leonard, P. M. Martin, J. R. Thompson, D. K. Christen R. Meng, I. Rusakova, C. W. Chu, and T. H. Johansen, Enhancement of flux pinning and critical currents in $\mathrm{YBa}_{2} \mathrm{Cu}_{3} \mathrm{O}_{7-\delta}$ films by nanoscale iridium pretreatment of substrate surfaces. J. Appl. Phys. 98, 114309 (2005).

[34] K. Matusmoto, T. Horide, K. Osamura, M. Mukaida,Y.Yoshida, A. Ichinose, and S. Horii, Enhancement of critical current density of YBCO films by introduction of 
artificial pinning centers due to the distributed nano-scaled $\mathrm{Y}_{2} \mathrm{O}_{3}$ islands on substrates. Physica C 412-414, 1267-1271 (2004).

[35] P. Mele, K. Matsumoto, T. Horide, O. Miura, A. Ichinose, M. Mukaida, Y. Yoshida, and S. Horii, Critical current enhancement in $\mathrm{PLD} \mathrm{YBa}{ }_{2} \mathrm{Cu}_{3} \mathrm{O}_{7-x}$ films using artificial pinning centers. Physica C 445-448, 648-651 (2006).

[36] J. C. Nie, H Yamasaki, H Yamada, Y Nakagawa, K Develos-Bagarinao and Y Mawatari, Evidence for c-axis correlated vortex pinning in $\mathrm{YBa}_{2} \mathrm{Cu}_{3} \mathrm{O}_{7-\delta}$ films on sapphire buffered with an atomically flat $\mathrm{CeO}_{2}$ layer having a high density of nanodots. Supercond. Sci. Technol. 17, 845-852 (2004).

[37] B. Maiorov, H. Wang, S. R. Foltyn, Y. Li, R. DePaula, L. Stan, P. N.Arendt, and L. Civale, Influence of naturally grown nanoparticles at the buffer layer in the flux pinning in $\mathrm{YBa}_{2} \mathrm{Cu}_{3} \mathrm{O}_{7}$ coated conductors. Supercond. Sci. Technol. 19, 891-895 (2006).

[38] V. F. Solovyov, H. JWiesmann, L. Wu, Q. Li, L. D. Cooley, M. Suenaga, B. Maiorov and L. Civale, High critical currents by isotropic magnetic-flux-pinning centres in a $3 \mu$ m-thick $\mathrm{YBa}_{2} \mathrm{Cu}_{3} \mathrm{O}_{7}$ superconducting coated conductor. Supercond. Sci. Technol. 20, L20-L23 (2007).

[39] T. Haugan, P. N. Barnes, R. Wheeler, F. Meisenkothen, and M. Sumption, Addition of nanoparticle dispersions to enhance flux pinning of the $\mathrm{YBa}_{2} \mathrm{Cu}_{3} \mathrm{O}_{7-x}$ superconductor. Nature 430, 867-870 (2004).

[40] T. Haugan, et al., Flux pinning strengths and mechanisms of YBCO with nanoparticle addition. Chapter in book Epitaxial Growth of Functional Oxides, 
edited by A. Goyal, Y. Kuo, O. Leonte, and W. Wong-Ng, (The Electrochemical Society Inc., Pennington NJ 2005), pages 359-366.

[41] J. L. MacManus-Driscoll, S. R. Foltyn, Q. X. Jia, H. Wang, A. Serquis, L. Civale, B. Maiorov, M. E. Hawley, M. P. Maley, and D. E. Peterson, Strongly enhanced current densities in superconducting coated conductors of $\mathrm{YBa}_{2} \mathrm{Cu}_{3} \mathrm{O}_{7-x}+$ $\mathrm{BaZrO}_{3}$. Nature Mater. 3, 439-443 (2004).

[42] A. A. Gapud, D. Kumar, S. K. Viswanathan, C. Cantoni, M. Varela, J. Abiade, S. J. Pennycook, and D. K. Christen, Enhancement of flux pinning in $\mathrm{YBa}_{2} \mathrm{Cu}_{3} \mathrm{O}_{7-\delta}$ thin films embedded with epitaxially grown $\mathrm{Y}_{2} \mathrm{O}_{3}$ nanostructures using a multilayering process. Supercond. Sci. Technol. 18, 1502-1505 (2005).

[43] J. Gutiérrez, A. Llordés, J. Gázquez, M. Gibert, N. Romá, S. Ricart, A. Pomar, F. Sandiumenge, N. Mestres, T. Puig and X. Obradors, Strong isotropic flux pinning in solution-derived $\mathrm{YBa}_{2} \mathrm{Cu}_{3} \mathrm{O}_{7-x}$ nanocomposite superconductor films. Nature Mater. 6, 367-373 (2007).

[44] J. Tersoff, Self-organized epitaxial growth of low-dimensional structures. Physica E 3, 89-91 (1998).

[45] C. Teichert, M.G. Legally, L.J. Peticolas, J.C. Bean, J. Tersoff, Stress-induced self-organization of nanoscale structures in SiGe/Si multilayer films. Phys. Rev. B 53, 16334-16337 (1996).

[46] A. Goyal, S. Kang, K. J. Leonard, P. M. Martin, A. A. Gapud, M. Varela, M. Paranthaman, A. O. Ijaduola, E. D. Specht, J. R. Thompson, D. K. Christen, S. J. Pennycook and F. A. List, Irradiation-free, columnar defects comprised of self- 
assembled nanodots and nanorods resulting in strongly enhanced flux-pinning in $\mathrm{YBa}_{2} \mathrm{Cu}_{3} \mathrm{O}_{7-\delta}$ films. Supercon. Sci. Technol. 18, 1533-1538 (2005).

[47] S. Kang, A. Goyal, J. Li, A. A. Gapud, P. M. Martin, L. Heatherly, J. R. Thompson, D. K. Christen, F. A. List, M. Paranthaman, and D. F. Lee, Highperformance high-T $T_{c}$ superconducting wires. Science 311, 1911-1914 (2006).

[48] Macmanus-Driscoll J L, Foltyn S R, Jia Q X, Wang H,Serquis A, Civale L, Maiorov B, Hawley M E, Maley M P and Peterson D E Nature Mater. 3, 439 (2004).

[49] A. Goyal, Engineered Columner Defects for Coated Conductors. Presented at the 2008 DOE Annual Peer Review on Superconductivity, available at http://www.energetics.com/supercon08/agenda.html

[50] Varanasi C V, Burke J, Wang H, Lee J H and Barnes P N Appl. Phys. Lett. 93, 092501 (2008).

[51] R. Weinstein, R. Sawh, A. Gandini, and D. Parks, Improved pinning by multiple inline damage. Supercond. Sci. Technol. 18, S188-S193 (2005).

[52] Q. Li, M. Suenaga, S. R. Foltyn, and H. Wang, $J_{c}(H)$ crossover in YBCO thick films and Bi2223/Ag tapes with columnar defects. IEEE Trans. on Appl. Supercon. 15, 2787-2789 (2005).

[53] S. R. Foltyn, H. Wang, L. Civale, Q. X. Jia, P. N. Arendt, B. Maiorov, Y. Li, M. P. Maley, and J. L. MacManus-Driscoll, Overcoming the barrier to $1000 \mathrm{~A} / \mathrm{cm}$ width superconducting coatings. Appl. Phys. Lett. 87, 162505 (2005).

[54] H. Zhou, B. Maiorov, H. Wang, J. L. MacManus-Driscoll, T. G. Holesinger, L. Civale, Q. X. Jia, and S. R. Foltyn, Improved microstructure and enhanced low- 
field Jc in $\left(Y_{0.67} E u_{0.33}\right) \mathrm{Ba}_{2} \mathrm{Cu}_{3} \mathrm{O}_{7-\delta}$ films. Supercond. Sci. Technol. 21, 025001025006 (2008).

[55] SungHun Wee, Amit Goyal, Yuri L. Zuev, and C. Cantoni, High performance superconducting wire in high applied magnetic fields via nanoscale defect engineering. Supercond. Sci. Technol. 21, 092001 (2008).

[56] 19. V. Selvamanickam, A. Guevara, Y. Zhang, I. Kesign, Y. Xie, G. Carota, Y. Chen, J. Dackow, Y. Zhang, Y. Zuev, C. Cantoni, A. Goyal, J. Coulter, and L. Civale Enhanced and Uniform in-Field Performance in Long (Gd,Y)-Ba-Cu-O Tapes with Zirconium Doping Fabricated by Metal Organic Chemical Vapor Deposition Superconductor Science \& Technology 23, 014014 (2010).

[57] Chen, Y.; Selvamanickam, V.; Zhang, Y.; Zuev, Y. L.; Cantoni,C.; Specht, E. D.; Paranthaman, M.; Aytug, T.; Goyal, A.; Lee, D. F. Enhanced Flux Pinning by $\mathrm{BaZrO}_{3}$ and $\left(\mathrm{Gd}, \mathrm{Y}_{2} \mathrm{O}_{3}\right.$ Nanostructures in Metal Organic Chemical Vapor Deposited GdYBCO High Temperature Superconductor Tapes. Appl. Phys. Lett. 94, 062513 (2008).

[58] Xiong, X.; Lenseth, K. P.; Reeves, J. L.; Rar, A.; Qiao, Y.; Schmidt, R. M.; Chen, Y.; Li, Y.; Xie, Y.; Selvamanickam, V. High Throughput Processing of LongLength IBAD MGO and Epi-Buffer Templates at Superpower. IEEE Trans. Appl. Supercond. 17, 3375 (2007).

[59] C. Cantoni, Y. Gao, S. H. Wee, E. D. Specht, J. Gazquez, J. Meng, S. J. Pennycook, and A. Goyal, Strain-Driven Oxygen Deficiency inSelf-Assembled, Nanostructured, Composite Oxide Films. ACS Nano 5, 4783 (2011). 
[60] Yu, H. H.; Shrotriya, P.; Gao, Y. F.; Kim, K.-S. Micro, Plasticity of Surface Steps under Adhesive Contact: Part I; Surface Yielding Controlled by SingleDislocation Nucleation. J. Mech. Phys. Solids 55, 489 (2007).

[61] Bozovic, I.; Logvenov, G.; Belca, I.; Narimbetov, B.; Sveklo, I. Epitaxial Strain and Superconductivity in $\mathrm{La}_{2-x} \mathrm{Sr}_{x} \mathrm{CuO} \mathrm{O}_{4}$ Thin Films. Phys. Rev. Lett. 89, 07001(4) (2002).

[62] Klenov, D. O.; Donner, W.; Foran, B.; Stemmer, S. Impact of Stress on Oxygen Vacancy Ordering in Epitaxial ( $\left.\mathrm{La}_{0.5} \mathrm{Sr}_{0.5}\right) \mathrm{CoO}_{3-\delta}$ Thin Films. Appl. Phys. Lett. 82,3427 (2003).

[63] Klie, R. F.; Buban, J. P.; Varela, M.; Franceschetti, A.; Jooss, C.; Zhu, Y.; Browning, N. D.; Pantelides, S. T.; Pennycook, S. J. Enhanced Current Transport at Grain Boundaries in High- $T_{c}$ Superconductors. Nature 435, 475 (2005).

[64] A. Llordés, A. Palau, J. Gázquez, M. Coll, R. Vlad, A. Pomar, J. Arbiol, R. Guzmán, S. Ye, V. Rouco, F. Sandiumenge, S. Ricart, T. Puig, M. Varela, Chateigner, J. Vanacken, D. J. Gutiérrez, V. Moshchalkov, G. Deutscher, C. Magen and X. Obradors, Nanoscale strain-induced pair suppression as a vortexpinning mechanism in hightemperature superconductors. Nat. Mat. 11, 329, (2011).

[65] Hammerl, G.; Schmehl, A.; Schulz, R. R.; Goetz, B.; Bielefeldt, H.; Schneider, C. W.; Hilgenkamp, H.; Mannhart, J. Enhanced Supercurrent Density in Polycrystalline $\mathrm{YBa}_{2} \mathrm{Cu}_{3} \mathrm{O}_{7-\delta}$ at $77 \mathrm{~K}$ from Calcium Doping of Grain Boundaries. Nature 407, 162 (200). 
[66] C. Cantoni, D. K. Christen, R. Feenstra, D. P. Norton, A. Goyal, G. W. Ownby, and D. M. Zehner, Reflection high-energy electron diffraction studies of epitaxial oxide seed-layer growth on rolling-assisted biaxially textured substrate Ni(001): The role of surface structure and chemistry. Appl. Phys. Lett. 79, 3077 (2001).

[67] C. Cantoni, D.K. Christen, L. Heatherly, M.M. Kowalewski, F.A. List, A. Goyal, G.W. Ownby, D.M. Zehner, B.W. Kang, and D.M. Kroeger, Quantification and control of the sulfur c $(2 \times 2)$ superstructure on $\{100\}<100>N i$ for optimization of $\mathrm{YSZ}, \mathrm{CeO}_{2}$ and $\mathrm{SrTiO}_{3}$ seed layers texture. J. Mater. Res. 17, 2549-2554 (2002). 


\section{Figure captions}

Fig. 1. Schematic of the two major technologies for HTS wires. The RABiTS architecture, (a), involves a sharply textured, single-crystal-like Ni-W alloy substrate on which $\mathrm{Y}_{2} \mathrm{O}_{3}$, YSZ, and $\mathrm{CeO}_{2}$ are epitaxial deposited to provide chemical and structural compatibility for the superconductor (YBCO) deposition. In the IBAD architecture, (b), the metal tape is polycrystalline Hastelloy. The texture is developed in the $\mathrm{MgO}$ film and transferred to the $\mathrm{YBCO}$ through a $\mathrm{LaMnO}_{3}$ layer. The $\mathrm{Al}_{2} \mathrm{O}_{3}$ layer serves as a diffusion barrier between oxides and metal. In both techniques the superconductor is capped with a $\sim 1 \mu m$-thick Ag layer followed by a $\sim 50 \mu m$-thick $\mathrm{Cu}$ layer for electrical, thermal, and environmental stability.

Fig. 2. Schematic diagram illustrating unpinned and pinned magnetic flux lines and random and extended defects. Columnar defects are capable of pinning the flux lines over the full length and, therefore, provide strong pinning effects.

Fig. 3. $\mathrm{J}_{\mathrm{c}}$ dependence on magnetic field for $\mathrm{YBCO}$ thin films on a single crystal, representing the intragranular $\mathrm{J}_{\mathrm{c}}$ (black line), and $\mathrm{SrTiO}_{3}$ bicrystals with [001] tilt or inplane tilt angles of $2^{\circ}, 4.5^{\circ}, 7^{\circ}, 15^{\circ}, 20^{\circ}$, and $24^{\circ}$. YBCO films grown on single crystal $\mathrm{SrTiO}_{3}$ are expected to show a mosaic spread of at least $1.8^{\circ}$ as a consequence of twinning domains [20]. The schematic to the right of the figure illustrates the direction of magnetic field, current, and pinning force in the measurements. 
Fig. 4. Angular dependence of $\mathrm{J}_{\mathrm{c}}$ for a $1.55 \mu \mathrm{m}$-thick $\mathrm{YBCO}$ film on $\mathrm{SrTiO}_{3}$ at $5 \mathrm{~T}$. Note that the largest current density is obtained when the field lies in the $a, b$ plane. The solid line represents the random pinning contribution. The various angular ranges of pinning are indicated. Courtesy L. Civale. The schematic on the right illustrates the geometry used in the measurements.

Fig. 5. Magnetic field dependence of $J_{\mathrm{c}}$. Three regions are distinguishable: a plateau at very small fields, a power low dependence at intermediate fields, and a rapid decay for fields larger than 1-2 $\mathrm{T}$.

Fig. 6. Nanosize defects typically observed in YBCO films for coated conductors: (a) antiphase boundaries (the crystal cells of two adjacent grains are out-of-registry along the c-axis); (b) stacking faults; (c) second phase spherical nanoparticles; (d) elongated, platelet-like intergrowths; (e) a-axis grains (the YBCO grows with the [100] perpendicular to the substrate); (f) low angle grain boundaries formed when slightly misoriented grains coalesce during growth.

Fig. 7. (a) $\mathrm{SrTiO}_{3}$ substrate decorated with $\mathrm{ZrO}_{2}$ nanodots deposited by PLD. (b) antiphase boundaries, stacking faults and plane buckling developed around a surface nanoparticle in the YBCO film grown on the decorated substrate shown in (a). In this particular sample $\mathrm{J}_{\mathrm{c}}$ was enhanced by a factor of 2 at $\mathrm{H}=1 \mathrm{~T}$ and $\mathrm{T}=40 \mathrm{~K}$ with respect to a control YBCO film on bare $\mathrm{SrtiO}_{3}$. 
Fig. 8. Cross-section high-resolution $\mathrm{STEM}$ of a $\mathrm{YBCO} / \mathrm{Y}_{2} \mathrm{O}_{3}$ composite film deposited with the multilayer approach. (a) Nanoparticles (indicated by arrows) are clearly discernible, with sizes ranging from 3 to $10 \mathrm{~nm}$ and about 5 to $20 \mathrm{~nm}$ apart. (b) Highresolution image of a $\mathrm{Y}_{2} \mathrm{O}_{3}$ nanoparticle, coherent with the $\mathrm{YBCO}$ matrix.

Fig. 9 Cross-section TEM image of a YBCO film on RABiTS with self-assembled $\mathrm{BaZrO}_{3}(\mathrm{BZO})$ nanodots and nanorods. The image shows that the $\mathrm{BZO}$ nanodots are aligned along the $c$ axis of YBCO and are about 2-3 $\mathrm{nm}$ in diameter.

Fig. 10. Low magnification, plan view TEM image of a YBCO film on RABiTS with self-assembled nanodots of $\mathrm{BaZrO}_{3}(\mathrm{BZO})$ showing some degree of order in the areal distribution of the nanocolumns (a). Corresponding autocorrelation function plot for an image derived from (a) by highlighting the BZO columns (b). The appearance of peaks around the central (auto-correlation) peak indicates a degree of ordering among the BZO columns.

Fig. 11. Z-contrast STEM image of a single BZO nanodot. Four misfit dislocations can be seen around the BZO nanodot. The extra semiplane in the edge dislocation cores are marked in blue. Different sets of planes in the YBCO and BZO are indicated.

Fig. 12. Left: $\log -\log$ plot of normalized $\mathrm{J}_{\mathrm{c}}$ as a function of applied field showing that the power low exponent in the relation $\mathrm{J}_{\mathrm{c}} \sim \mathrm{H}^{-\alpha}$ is lower in the $\mathrm{YBCO}$ film with selfassembled nanodots than for the control, undoped YBCO film. Right: angular 
dependence of $\mathrm{J}_{\mathrm{c}}$ at $77 \mathrm{~K}, 1 \mathrm{~T}$ for an undoped YBCO film on RABiTS compared to a YBCO film with 2 vol. \% BZO nanodots. Significant enhancement of $\mathrm{J}_{\mathrm{c}}$ is seen at all angles, particularly at $\mathrm{H} / / c\left(\right.$ angle $\left.=0^{\circ}\right)$. Both superconducting films have a thickness of $0.2 \mu \mathrm{m}$.

Fig. 13. Cross-section HRTEM images of BZO nanocolumns in a 4- $\mu m$-thick YBCO film. The columns extend through the entire thickness of the film and show small deviations in their direction from the $\mathrm{YBCO} c$ direction.

Fig. 14. (a) TEM micrograph illustrating the morphology of a 4 vol \% BZO nanocolumn array embedded in the YBCO matrix. (b) Volumetric average of the out-of-plane strain $\left(\varepsilon_{33} / f_{0} ; f_{0}=\right.$ lattice misfit) calculated through finite element method for the case $d / L=1$ (d) Strain versus BZO concentration as calculated by the finite element method for the cases $\mathrm{d} / \mathrm{L}=20$ coherent interface (triangles), $d / L=1$ coherent interface (black dots), $d / L=1$ incoherent interface (circles), and measured by X-ray diffraction for PLD films (open squares) and MOCVD films (filled squares). Adapted from ref. 57.

Fig 15. (a) High-resolution Z-STEM image of a sectioned BZO nanocolumn.

dashed circles indicate pure tilt dislocations; white arrows indicate misfit dislocations. (d) Comparison between analytically calculated strain (dots) and experimentally derived strain (squares). Adapted from ref. 57. 
Fig 16. (a) EELS maps of O-K, Ba-M4,5, Cu-L2,3, and Gd-M4,5. The top map is the signal from the ADF detector acquired simultaneously to each spectrum. (b) Comparison of $\mathrm{Cu}-\mathrm{L}_{2,3}$ edges after removal of plural scattering by deconvolution for the regions labeled 1 and 2 in (b).

Fig. 17. Schematic model for the nucleation of $\mathrm{CeO}_{2}$ and $\mathrm{STO}$ on a (001) Ni surface with chemisorbed $c(2 \times 2)$ sulfur superstructure. The dashed lines indicate correspondence between oxygen sites in (001) planes of the seed layer and sulfur sites on the Ni surface. The seed layer cations impinging on the Ni surface bond easily to the sulfur atoms present on the metal surface, promoting the (001) orientation of the growing film. In the STO case, there is a 1:1 correspondence between oxygen atoms in the $\mathrm{SrO}$ plane and sulfur atoms on the Ni surface. Therefore, it is plausible that Ti ions initially bond to the S surface atoms to form the first $\mathrm{TiO}_{2}$ plane of the $\mathrm{STO}$ structure. In the $\mathrm{CeO}_{2}$ case, two of four oxygen ions/unit cell match the sulfur atoms of the $c(2 \times 2)$. During nucleation of $\mathrm{CeO}_{2}$, oxygen atoms may fill in the empty fourfold $\mathrm{Ni}$ hollows and the Ce cations subsequently bond to the template formed by $\mathrm{S}$ and $\mathrm{O}$. 


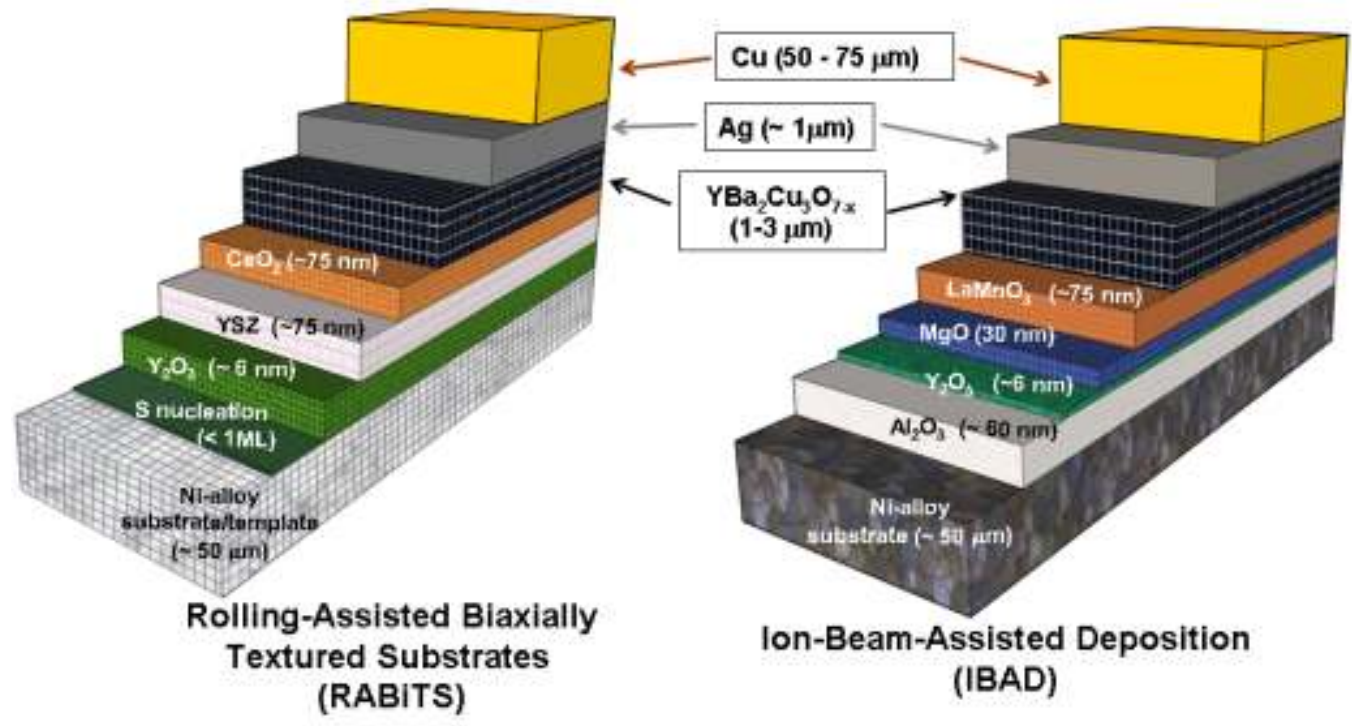

Fig. 1

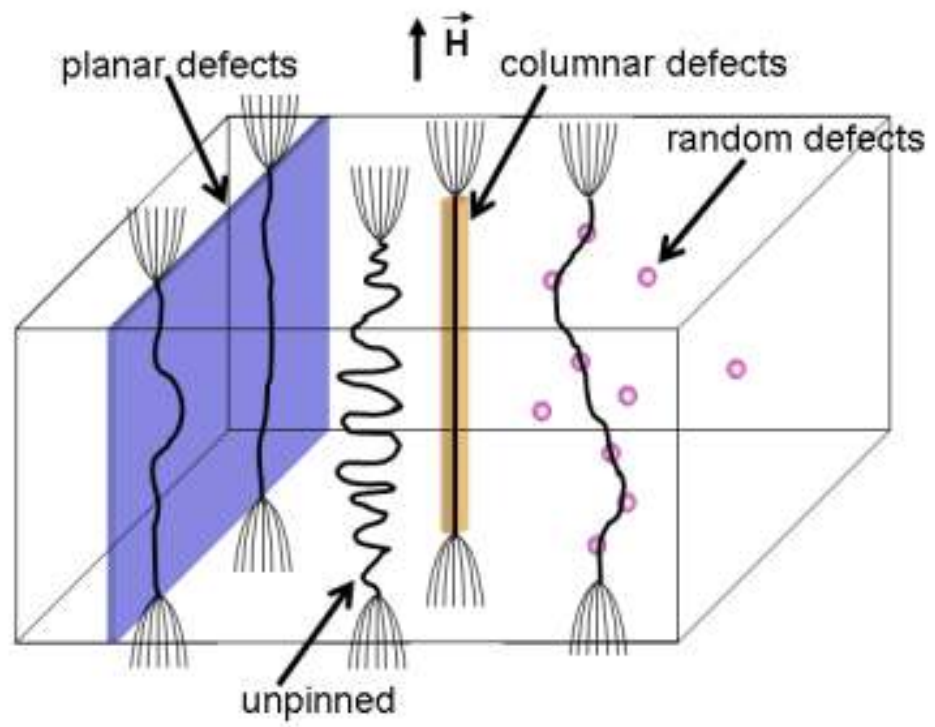

Fig. 2 


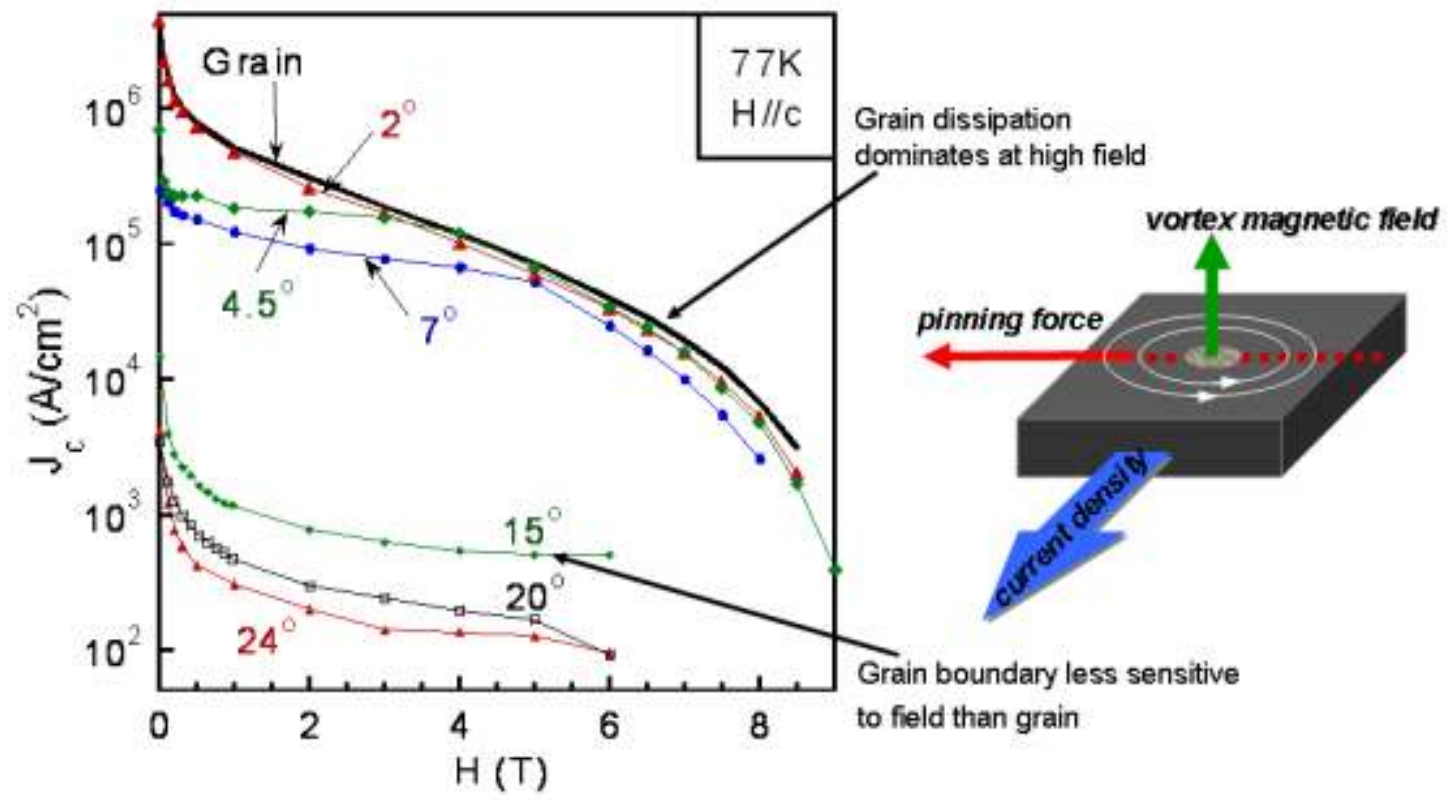

Fig. 3
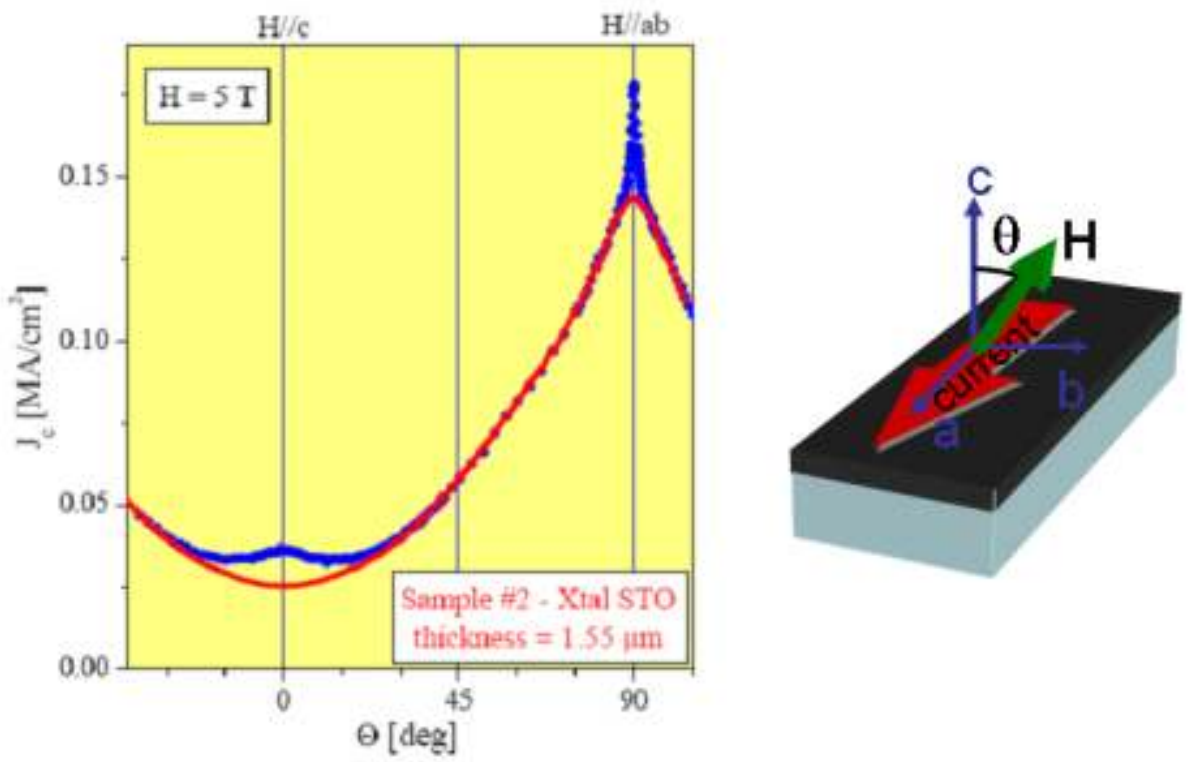

Fig. 4 


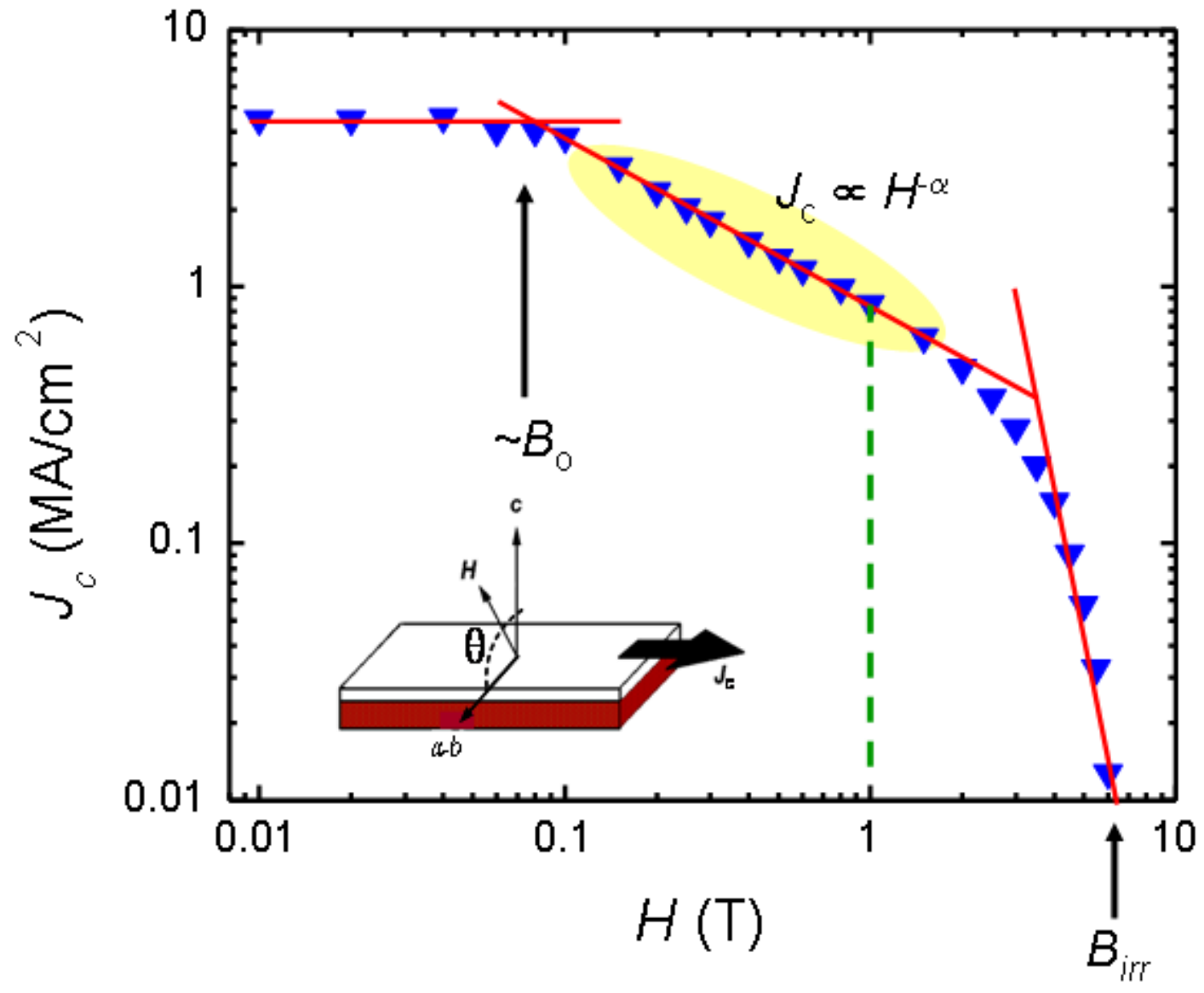

Fig. 5 

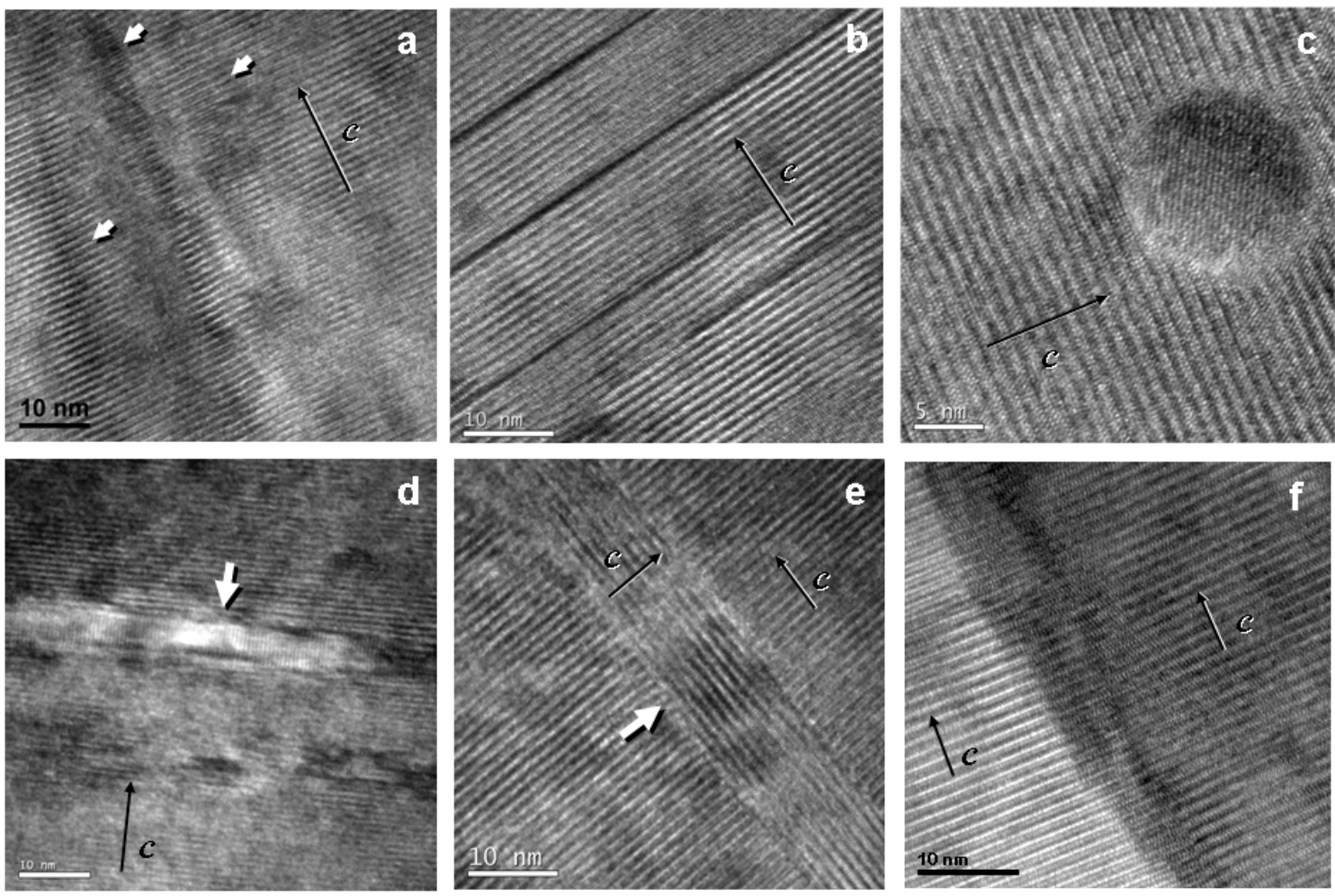

Fig. 6
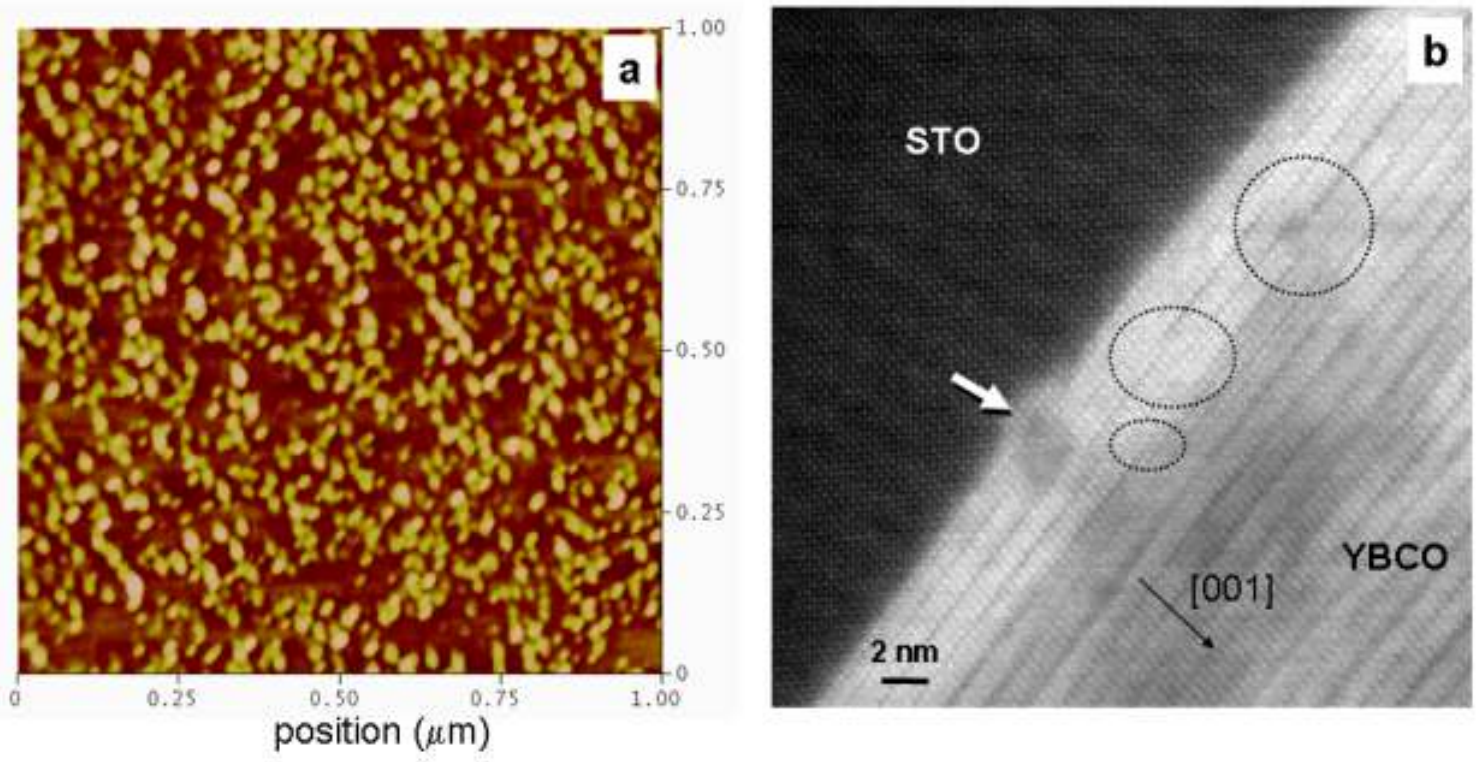

Fig. 7 

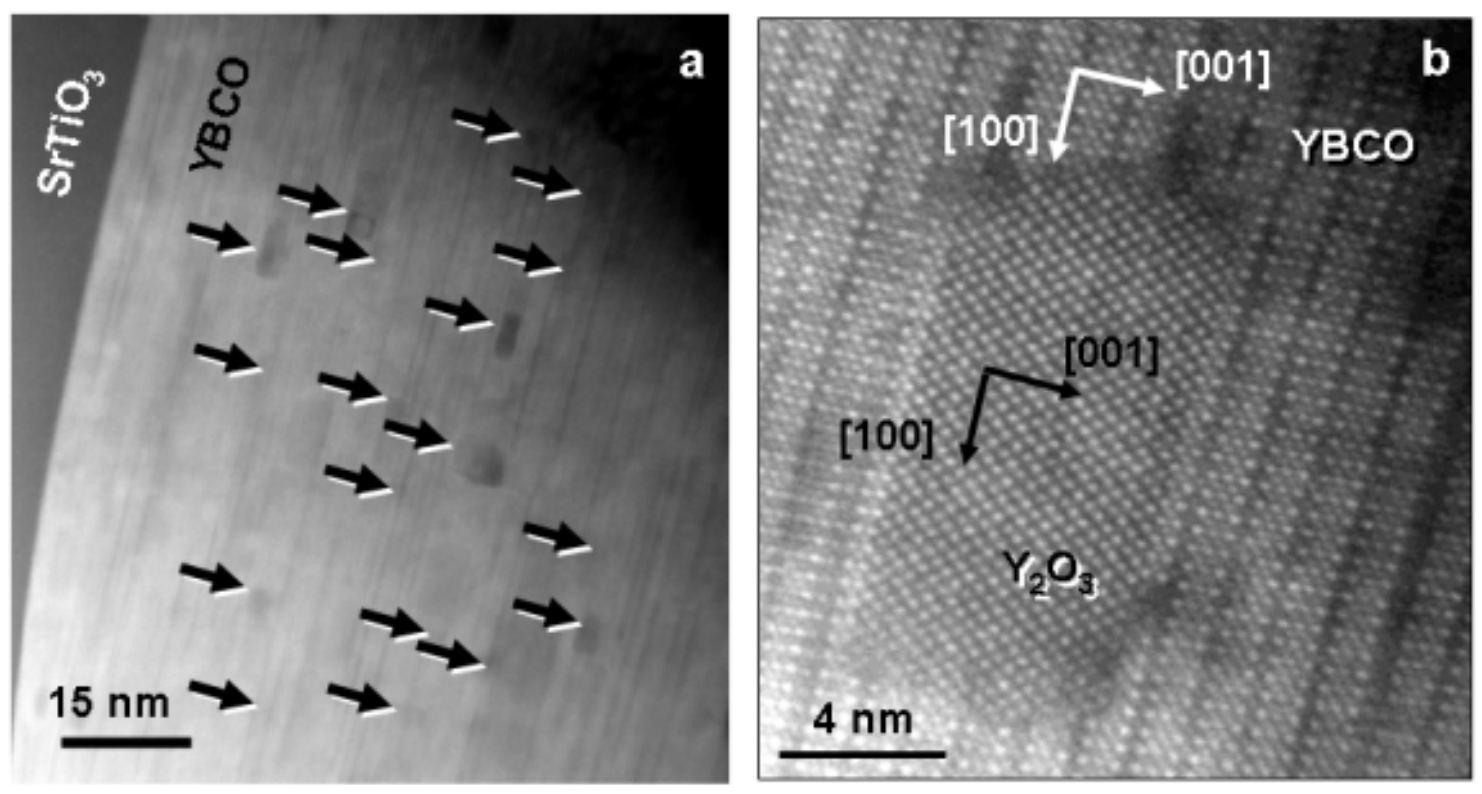

Fig. 8 


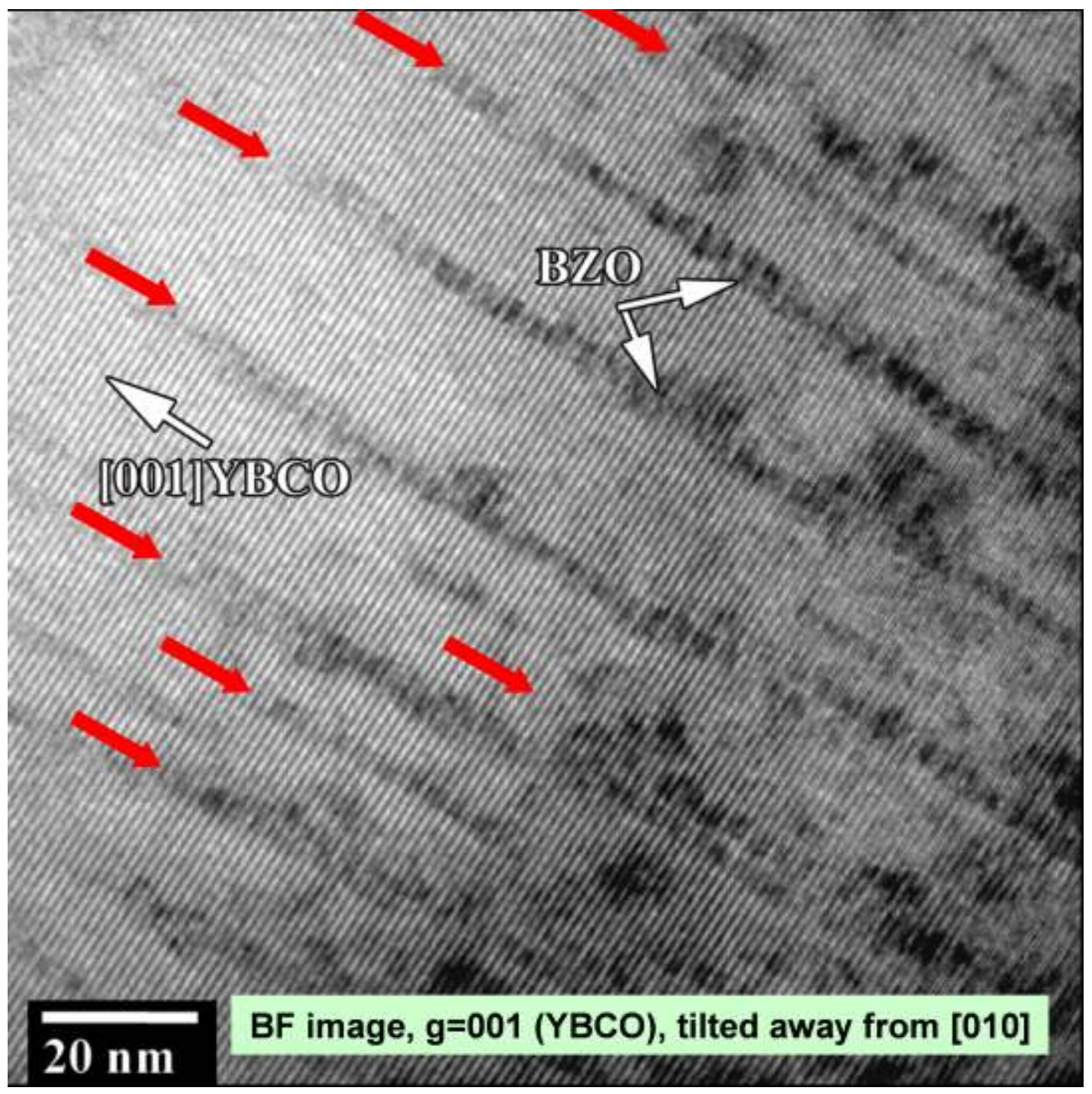

Fig. 9 

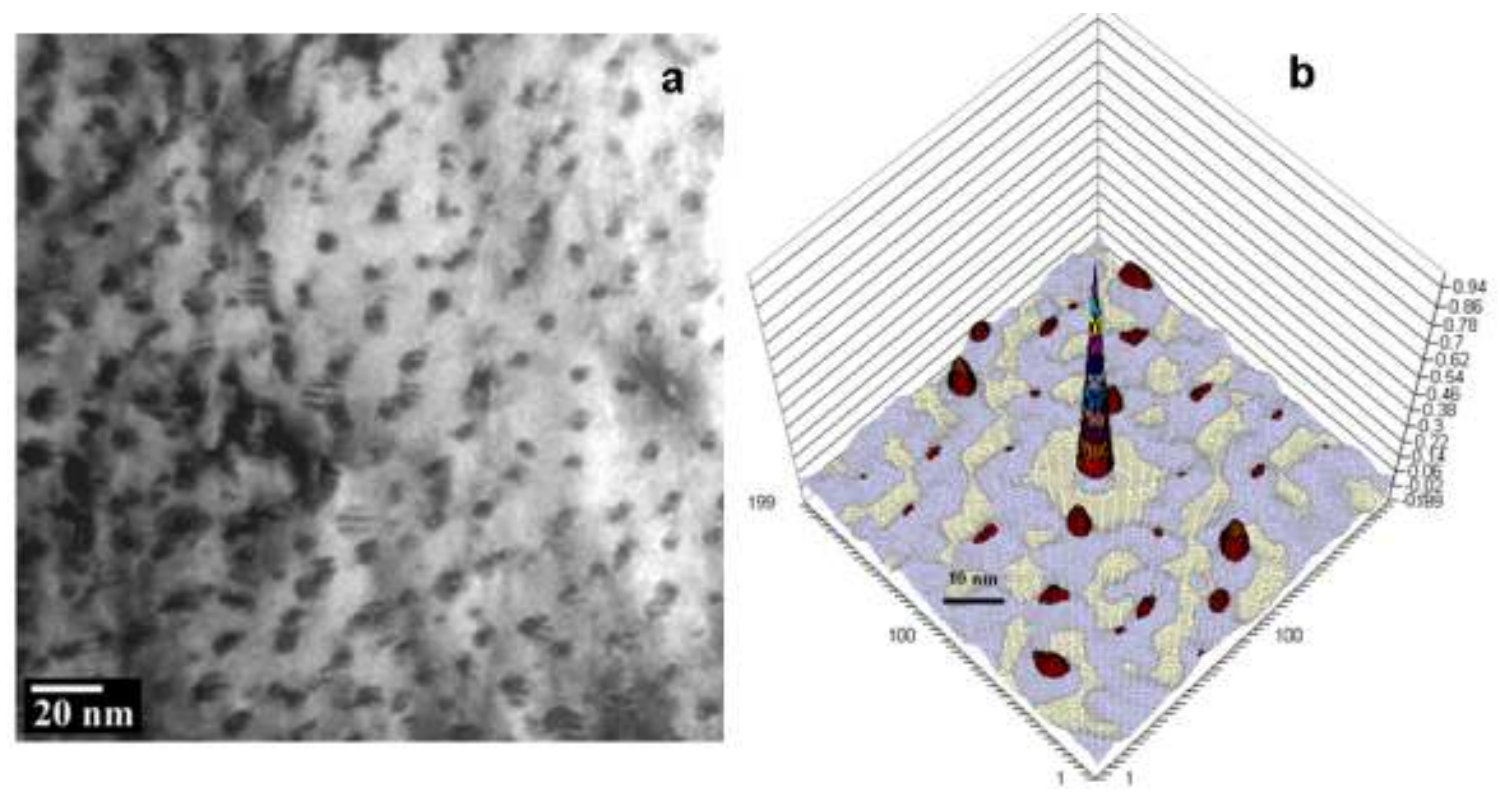

Fig. 10 


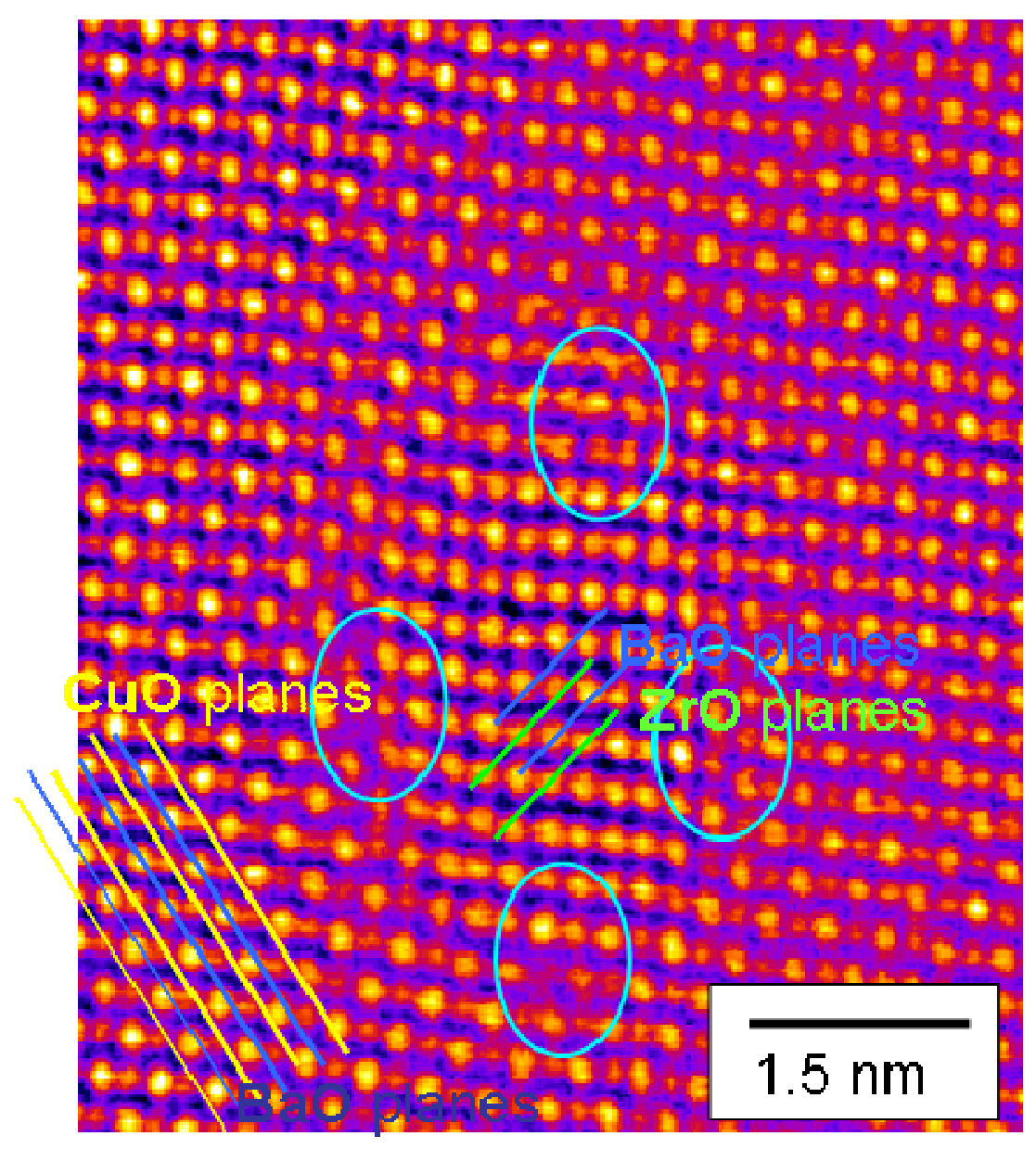

Fig. 11 
a

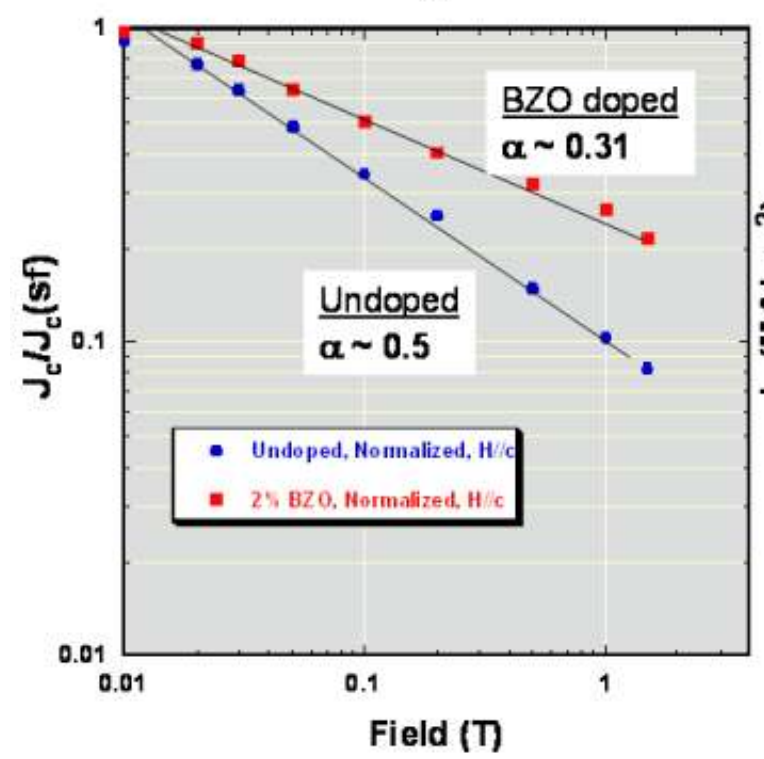

b

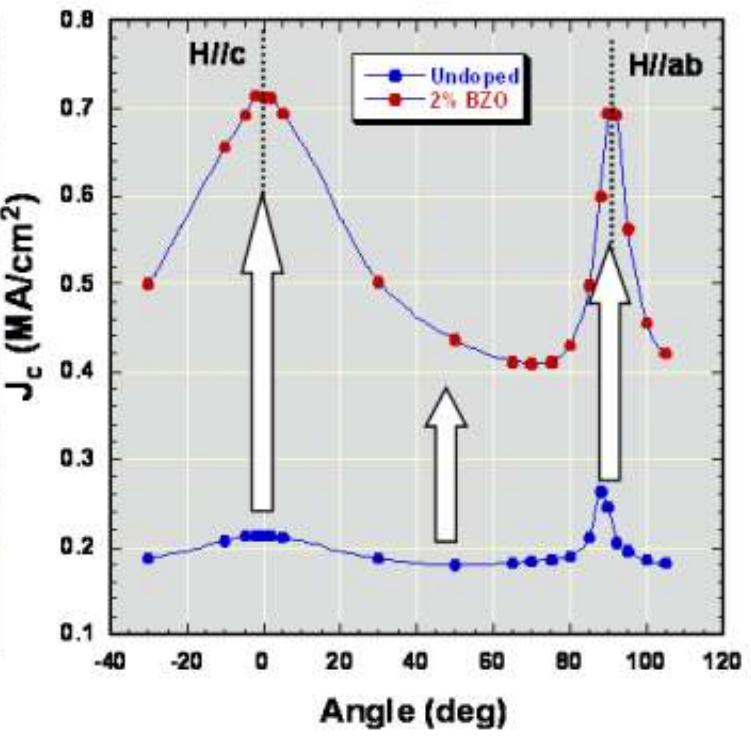

Fig. 12
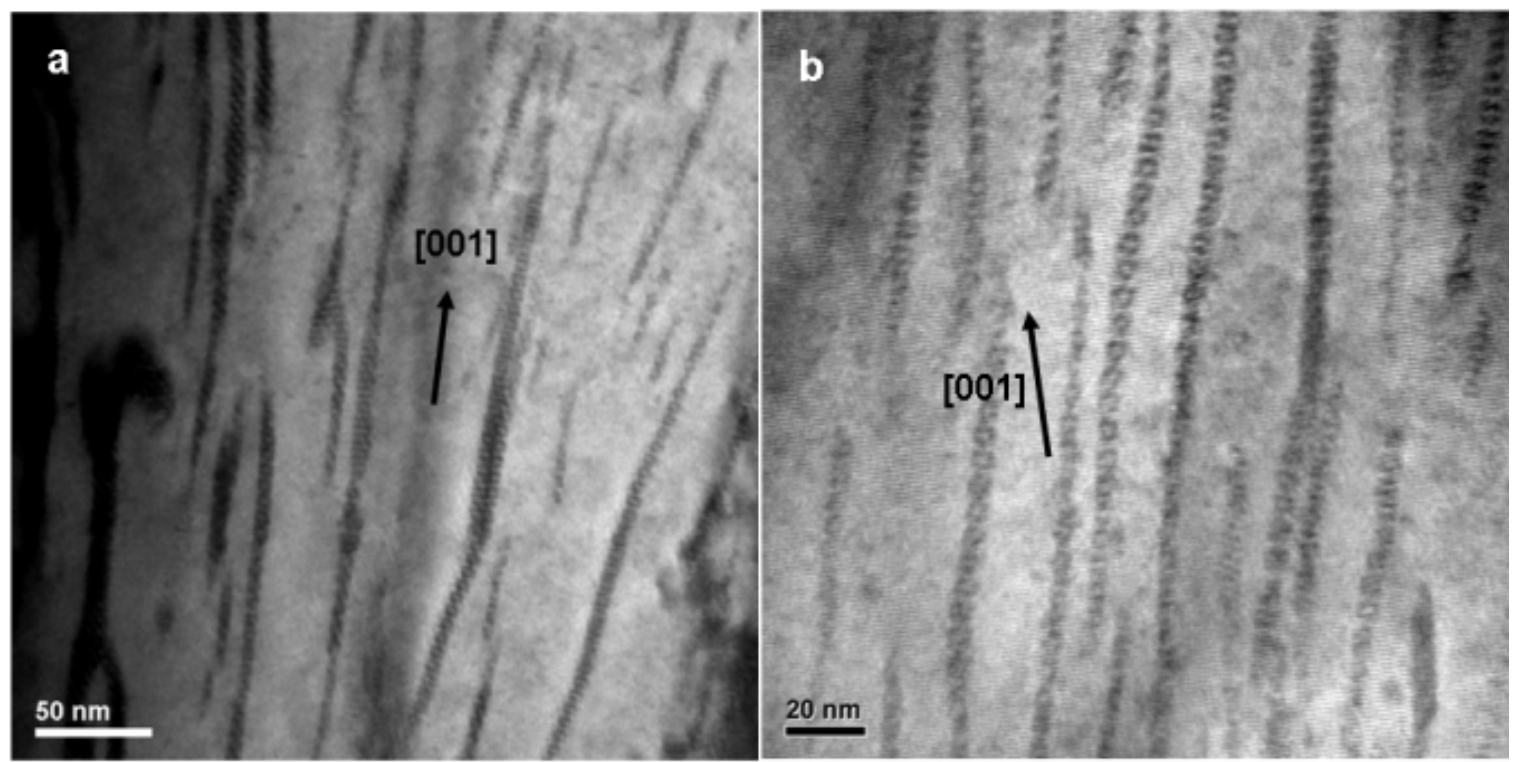

Fig. 13 
(a)
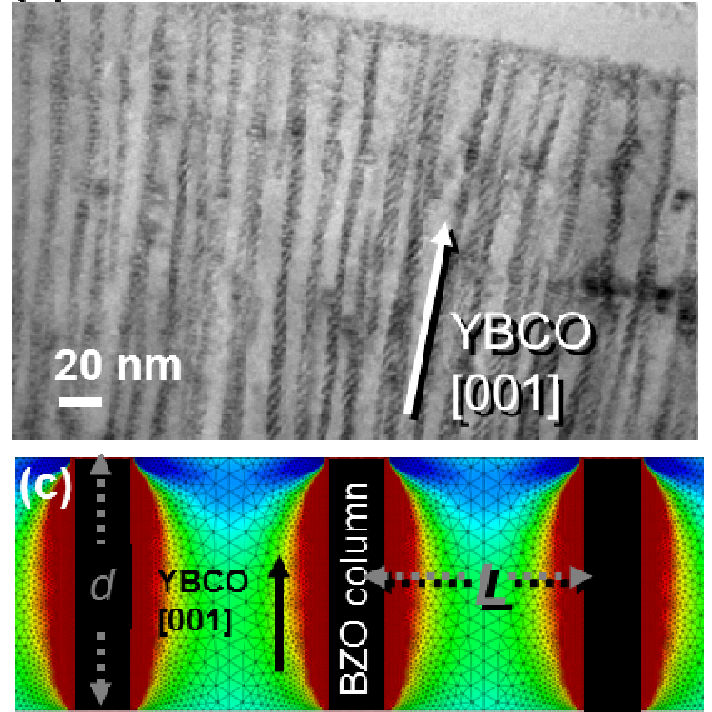

(b)

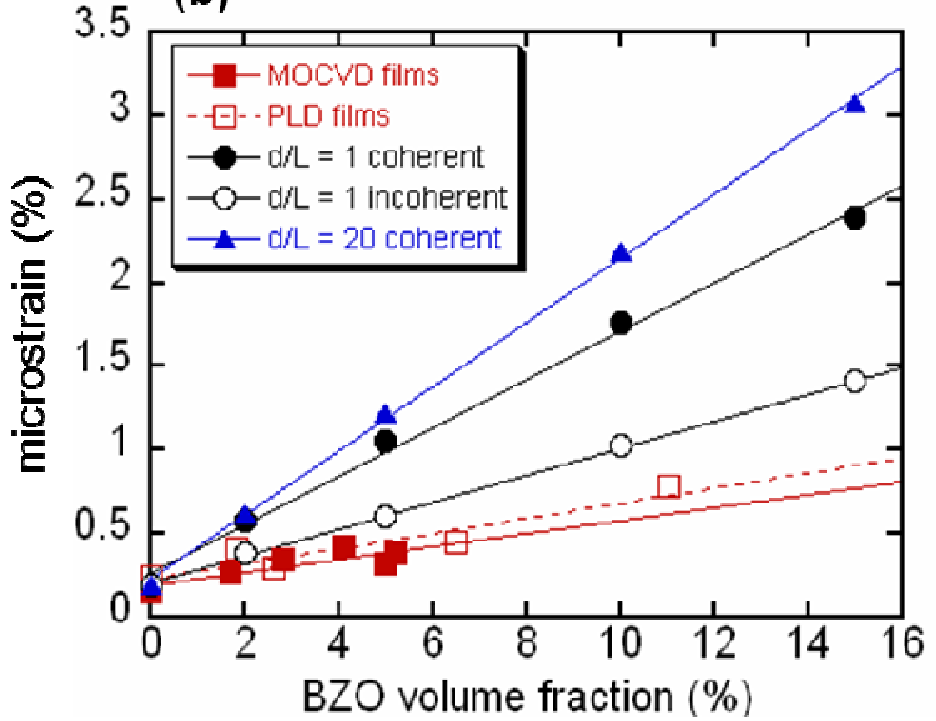

Fig 14 

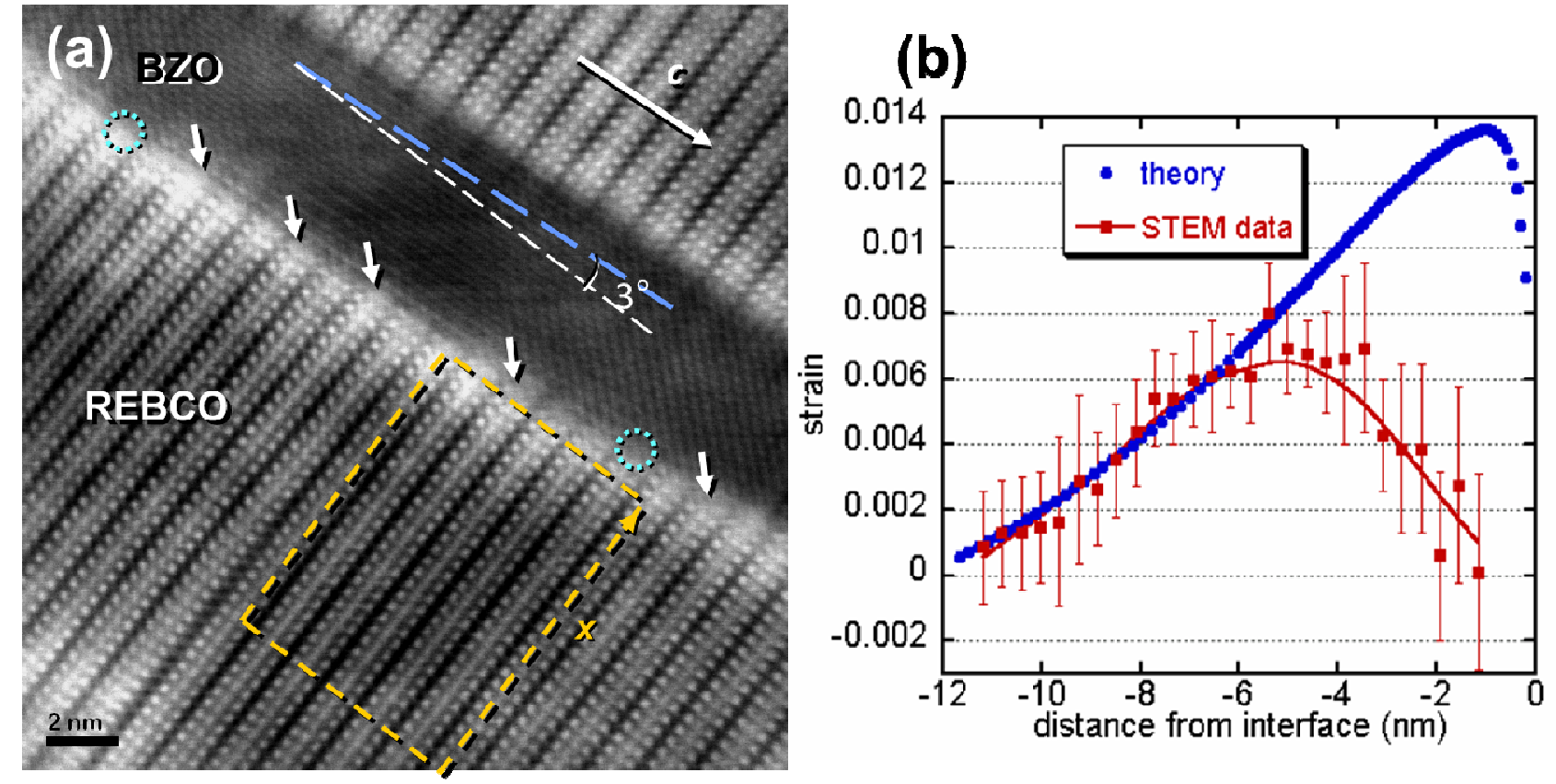

Fig. 15

\section{nanorod}
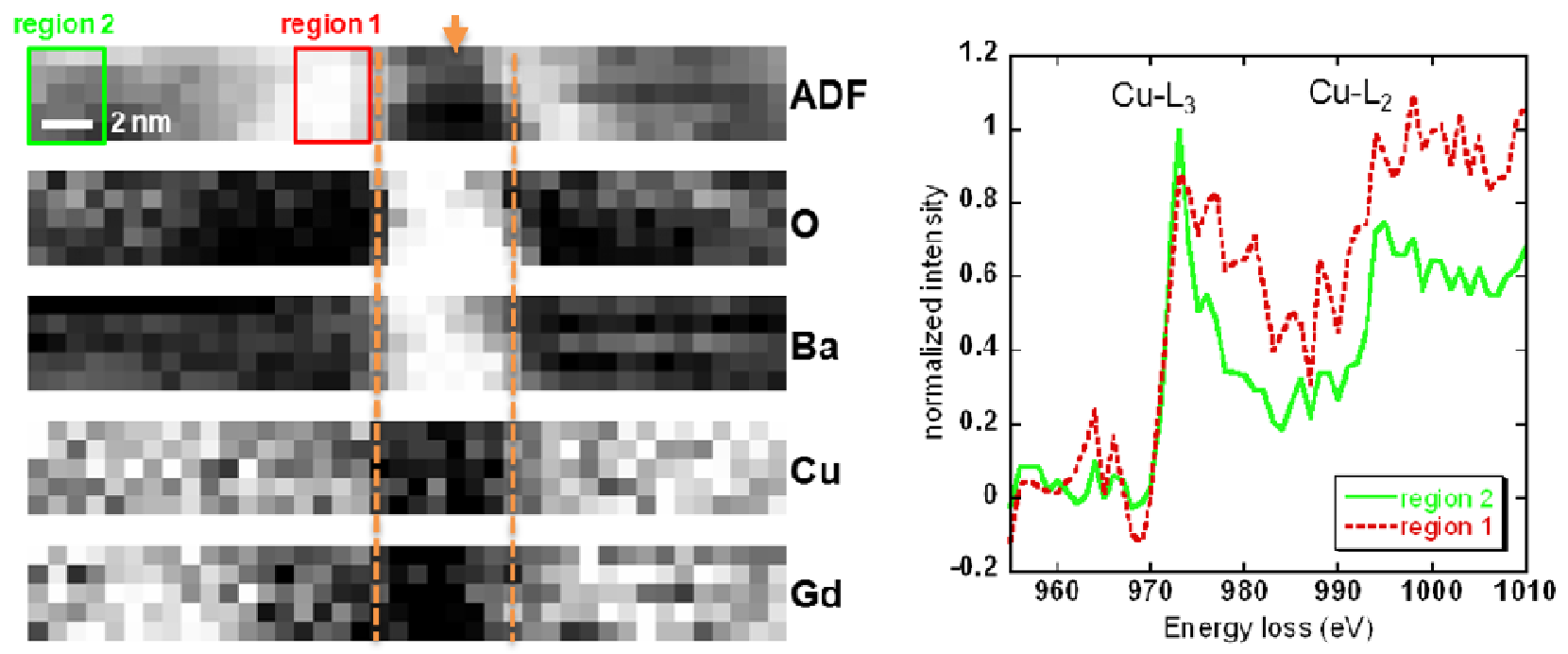

Fig 16 


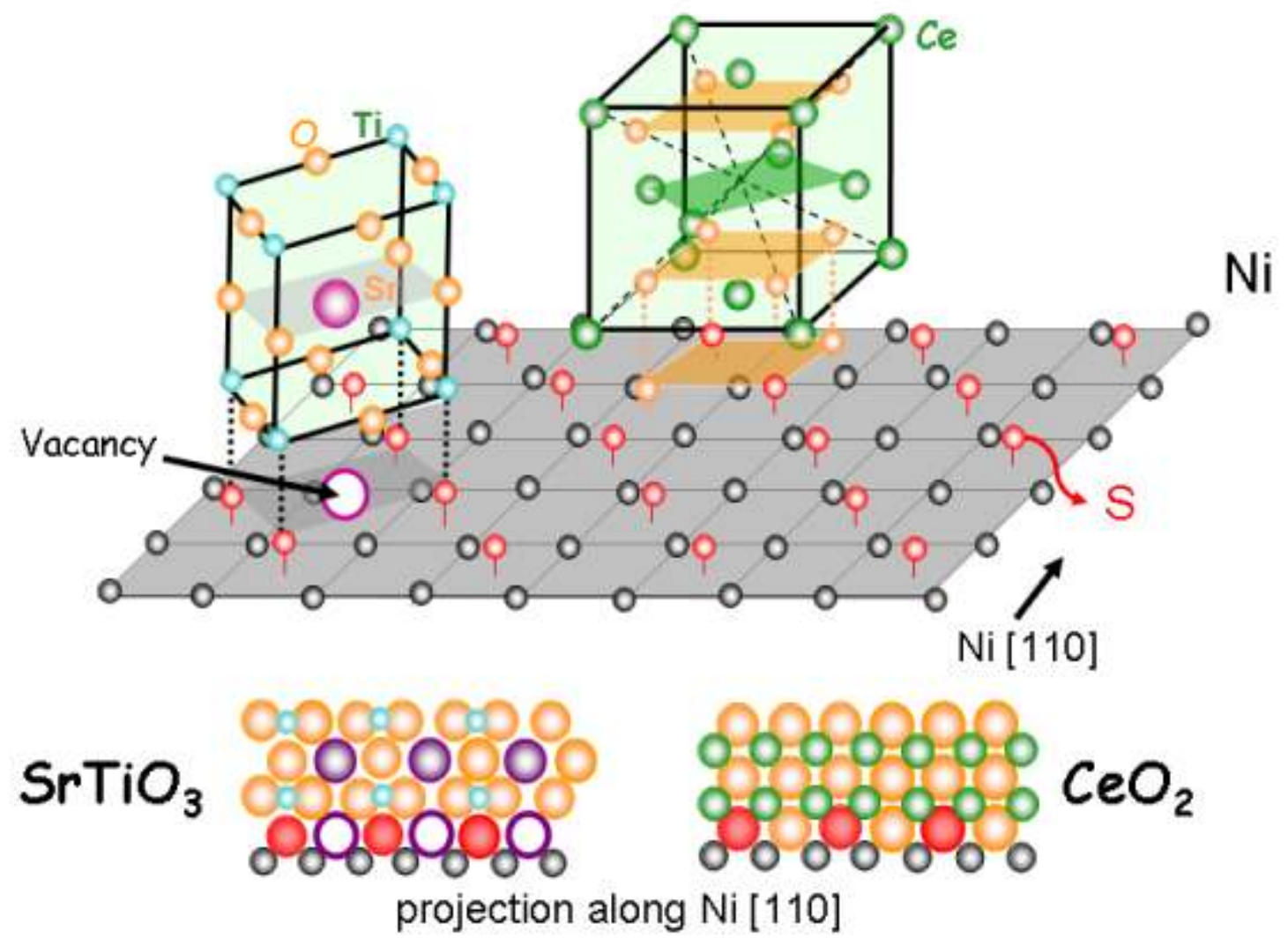

Fig. 17 